\title{
Analyzing the competition of gamma rhythms with delayed pulse-coupled oscillators in phase representation
}

\author{
Atthaphon Viriyopase, ${ }^{1,2,3}$ Raoul-Martin Memmesheimer, ${ }^{1,3,4,5,6}$ and Stan Gielen ${ }^{1,2}$ \\ ${ }^{1}$ Donders Institute for Brain, Cognition, and Behaviour, Radboud University Nijmegen, Nijmegen, The Netherlands \\ ${ }^{2}$ Department of Biophysics, Faculty of Science, Radboud University Nijmegen, Nijmegen, The Netherlands \\ ${ }^{3}$ Department of Neuroinformatics, Faculty of Science, Radboud University Nijmegen, Nijmegen, The Netherlands \\ ${ }^{4}$ Center for Theoretical Neuroscience, Columbia University, New York, New York 10027, USA \\ ${ }^{5}$ FIAS-Frankfurt Institute for Advanced Studies, Frankfurt am Main, Germany \\ ${ }^{6}$ Neural Network Dynamics and Computation, Institute of Genetics, University of Bonn, Bonn, Germany
}

(Received 3 July 2018; published xxxxxx)

\begin{abstract}
Networks of neurons can generate oscillatory activity as result of various types of coupling that lead to synchronization. A prominent type of oscillatory activity is gamma (30-80 Hz) rhythms, which may play an important role in neuronal information processing. Two mechanisms have mainly been proposed for their generation: (1) interneuron network gamma (ING) and (2) pyramidal-interneuron network gamma (PING). In vitro and in vivo experiments have shown that both mechanisms can exist in the same cortical circuits. This raises the questions: How do ING and PING interact when both can in principle occur? Are the network dynamics a superposition, or do ING and PING interact in a nonlinear way and if so, how? In this article, we first generalize the phase representation for nonlinear one-dimensional pulse coupled oscillators as introduced by Mirollo and Strogatz to type II oscillators whose phase response curve (PRC) has zero crossings. We then give a full theoretical analysis for the regular gamma-like oscillations of simple networks consisting of two neural oscillators, an "E neuron" mimicking a synchronized group of pyramidal cells, and an "I neuron" representing such a group of interneurons. Motivated by experimental findings, we choose the E neuron to have a type I PRC [leaky integrate-and-fire (LIF) neuron], while the I neuron has either a type I or type II PRC (LIF or "sine" neuron). The phase representation allows us to define in a simple manner scenarios of interaction between the two neurons, which are independent of the types and the details of the neuron models. The presence of delay in the couplings leads to an increased number of scenarios relevant for gamma-like oscillatory patterns. We analytically derive the set of such scenarios and describe their occurrence in terms of parameter values such as synaptic connectivity and drive to the E and I neurons. The networks can be tuned to oscillate in an ING or PING mode. We focus particularly on the transition region where both rhythms compete to govern the network dynamics and compare with oscillations in reduced networks, which can only generate either ING or PING. Our analytically derived oscillation frequency diagrams indicate that except for small coexistence regions, the networks generate ING if the oscillation frequency of the reduced ING network exceeds that of the reduced PING network, and vice versa. For networks with the LIF I neuron, the network oscillation frequency slightly exceeds the frequencies of corresponding reduced networks, while it lies between them for networks with the sine I neuron. In networks oscillating in ING (PING) mode, the oscillation frequency responds faster to changes in the drive to the I (E) neuron than to changes in the drive to the $\mathrm{E}$ (I) neuron. This finding suggests a method to analyze which mechanism governs an observed network oscillation. Notably, also when the network operates in ING mode, the E neuron can spike before the I neuron such that relative spike times of the pyramidal cells and the interneurons alone are not conclusive for distinguishing ING and PING.
\end{abstract}

DOI: 10.1103/PhysRevE.00.002200

\section{INTRODUCTION}

Many processes in biology, physics, chemistry, and engineering have an oscillatory character. Regular oscillations on a limit cycle can be described by a single variable, the phase, which characterizes the time needed to reach the current state due to unperturbed dynamics when starting from some specified "reset" point on the cycle (e.g., [1,2]). If an oscillator receives inputs in the form of pulses and an input-induced perturbation from the limit cycle relaxes back sufficiently quickly (i.e., before the next input arrives), the system's dynamics can be characterized by the phase together with a function telling how the phase changes in response to an input 54 pulse: the phase response curve (PRC) or the phase transition 55 curve or transfer function $[1,3,4]$. This phase representation has 56 been widely used to investigate network dynamics, especially 57 synchronization and locking phenomena, in areas of science 58 as diverse as neural circuits [5-8], technical networks [9,10], 59 and insect behavior [4,11].

A particularly simple type of oscillator is given by a hybrid dynamical system whose state variable follows some 62 one-dimensional, possibly nonlinear continuous dynamics, 63 periodically reaches a threshold, and is then reset [12]. A rich 64 source of such oscillators is the reduction of spiking neurons 65 
66 to integrate-and-fire type neuron models [13-15]: Biological 67 neurons possess a complicated branched structure with protru-

68 sions of different function and many slow and fast degrees

69 of freedom associated with the resulting compartments. In

integrate-and-fire type neuron models, this spatial structure is

71 reduced to a single compartment "point neuron" and the high-

dimensional dynamics are reduced to one degree of freedom,

interpreted as the membrane potential $[1,16]$. Integrate-and-fire

74 type neurons interact with pulses, mimicking spikes or action

potentials; these are sent when the neuron is reset and are

received by postsynaptic neurons often after some delay. In

this article, we consider networks of two integrate-and-fire type

neurons in phase representation to investigate the competition

between mechanisms that are widely assumed to underlie

oscillations in biological neural networks. Each integrate-and-

fire type neuron thereby represents a synchronized population of neurons.

Oscillations in biological neural networks may be important

for information processing $[17,18]$. One hypothesis is that they

may coordinate precise spike sending of neurons and lead

86 to synchronous spiking of neural populations [19]. Indeed,

87 experiments have found examples of highly synchronous

38 spiking associated with strong oscillations [20] and the timing

89 of individual spikes relative to a global oscillation's phase can

90 carry important information [19,21-24]. Receiving neurons, in

91 turn, can be highly sensitive to coincident input; in particular,

92 types of synaptic plasticity depend on the timing of spikes [25].

93 Under high-input conditions the spike-generating mechanism

94 can adaptively enhance the sensitivity to synchronous input

95 while simultaneously decreasing the sensitivity to tempo-

rally uncorrelated inputs [26]. Further, oscillatory modulation

7 of the membrane potential, for example, by input from a

synchronously firing population of neurons, can provide a

precise temporal window for the integration of synaptic inputs,

favoring inputs arriving precisely at certain times [27,28].

The "communication through coherence" hypothesis suggests

that this promotes information transmission between coher-

ently oscillating neuron populations in different brain areas

and allows us to focus on attended stimuli [29-32]. Higher

frequency oscillations may support propagation and selection

of information within areas [33,34]. Oscillation coordinated

synchronous spiking across different neuron populations may

also allow us to bind different features of a stimulus into a

coherent percept [35-39] and generally parse and separate

information into chunks of different length [22,40,41].

In the current article, we will focus on gamma (30-

$80 \mathrm{~Hz}$ ) oscillations. These are prominent oscillations, which have been linked to input selectivity $[30,42]$, spike-phase encoding [19,43], feature binding [35], as well as to storage and retrieval of information [40,41]. Mainly two mechanisms have been proposed to underlie gamma oscillations [44-46]. Both involve populations of excitatory pyramidal cells ( $E$ cells) and inhibitory interneurons (I cells). Tonic excitation of the interneurons, e.g., due to averaging slow excitatory input, can give rise to interneuron network gamma (ING) [47-52]: Imagine, by chance at some point more I cells spike and generate increased inhibition. This hinders the other I 123 cells from spiking before the ones that have just spiked have 124 recovered, and recruits them into synchrony such that a rhythm 125 emerges [53]. The I cells undergo a cycle of enhanced spiking activity, resulting in increased recurrent inhibition within 126 the population, subsequently decreased activity, followed by ${ }_{127}$ recovery from inhibition and again enhanced spiking. The ${ }_{128}$ resulting periodically increased inhibition generates rhythmic ${ }_{129}$ spiking in connected E cells. Pyramidal-interneuron network ${ }_{130}$ gamma (PING) is mediated by interacting populations of ${ }_{131}$ E cells and I cells [51,54,55]. Imagine, by chance at some ${ }_{132}$ point more E cells spike. The I cells respond to the increased ${ }_{133}$ excitatory input from the E cells by increasing their spiking. ${ }_{134}$ The resulting increased inhibitory input in turn hinders spiking 135 in the E cells, such that their activity goes down. The lack ${ }_{136}$ of excitatory input leads to a decrease of I-cell activity, such ${ }_{137}$ that the E cells can recover from inhibition and generate 138 increased spiking, which completes the cycle. To summarize, 139 ING relies crucially on mutual inhibition generated by the I ${ }_{140}$ cells among each other, while PING relies crucially on the 141 $E \rightarrow I$ connections and the inhibitory feedback to the E cells. ${ }_{142}$ In model networks, there can be a sharp boundary in parameter ${ }_{143}$ space between the regime in which the I cells have weak enough 144 drive for PING, and the ING regime in which the drive to the I ${ }_{145}$ cells is so large that they fire without being prompted by the E ${ }_{146}$ cells [56]. However, recent studies have shown that this sharp ${ }_{147}$ transition may be a simplification [57] and we highlighted in 148 Ref. [58] that there are two-neuron systems that can generate 149 ING as well as PING, depending on the initial conditions. 150

Using computer simulations of larger networks, in Ref. [58] ${ }_{151}$ we have shown that in the range of parameter space where 152 ING and PING may in principle be expected to exist, both 153 mechanisms compete such that the mechanism generating 154 the higher oscillation frequency "wins"; i.e., the mechanism 155 with the higher frequency determines the frequency of the 156 network oscillation and suppresses the other one. In the ${ }_{157}$ current article we provide a theoretical analysis of the finding, 158 using simplified networks of two oscillating integrate-and-fire 159 type neurons. The simplified system allows us to analytically 160 study the interactions between ING and PING and to better 161 understand their consequences for oscillations in networks of 162 interacting E cells and I cells. The analytically tractable model ${ }_{163}$ consists of an E neuron, which belongs to the category of type 164 I neurons, and an I neuron, which can be either type I or type 165 II. For type I neurons an excitatory input always advances 166 the next spike; the PRC is entirely positive. In contrast, an ${ }_{167}$ excitatory input arriving at a type II neuron can also delay ${ }_{168}$ the next spike; the PRC is partially negative [1,59]. Indeed, 169 there is experimental evidence that I cells involved in gamma 170 oscillations may belong to the category of type II neurons 171 [60-62].

We consider current-based integrate-and-fire neurons, 173 where the currents have infinitesimally short temporal dura- 174 tion. The latter implies that the membrane potential responds 175 in jumplike manner to the input, the former that the height of the 176 jump is independent of the membrane potential. Note that also 177 some conductance-based and more general models can be cast 178 into this form by a transformation of variables [63,64]. For type 179 I neurons, where an excitatory jump (towards the membrane 180 potential threshold) always advances the phase, a phase repre- 181 sentation has been derived in Refs. [4,65]. We adopt this phase ${ }_{182}$ representation for our type I neurons since the linearization 183 of the free dynamics strongly simplifies the analytical study 184 of the system and since the phase representation allows for 185 
186 simple and fast event-based numerical simulations. To be able 187 to study networks with type II interneurons in the same way, we 188 derive a generalized phase representation, which is applicable 89 to neurons of this type. For this, we assume that an infinitesimal 90 phase response curve (iPRC) of type II is given, and we derive 191 the corresponding membrane potential dynamics as well as the 192 PRC.

193 The article is structured as follows: Section II is dedicated to 194 the standard phase representation of a one-dimensional oscil195 lator, its derivation from the free dynamics, and its application 196 to the leaky integrate-and-fire (LIF) neuron, which is the type I 197 neuron model that we use throughout the article. In Sec. III, we 198 derive the phase representation of one-dimensional oscillators 199 of type II, where the iPRC can change sign. We apply the 200 scheme to derive the "sine neuron," the type II neuron model 201 that we use throughout the article. The Appendix compares 202 this neuron with the radial isochron clock, an oscillator model 203 that has the same iPRC. In Sec. IV, we consider delayed pulse204 coupled networks of two model neurons and show the ways in which they interact depending on their phase difference. This yields a representation of the dynamics in terms of iteration maps whose fixed points yield the regular oscillations that we study in Sec. V. Section VI is dedicated to the competition and coexistence of the ING and PING oscillation mechanisms. We conclude with a discussion in Sec. VII, which puts our findings in context to the existing literature and our previous larger scale simulation studies [58]. We note that in Ref. [58] we summarized, displayed, and discussed some of the results of the current article.

\section{PHASE REPRESENTATION OF TYPE I ONE-DIMENSIONAL OSCILLATORS}

\section{A. General theory}

In the following, we review the standard phase representation of one-dimensional oscillators coupled by infinitesimally short pulsed interactions proposed in Refs. [4,8,65], as needed for the purposes of the present article. For a more general derivation and discussion, see [65].

A one-dimensional neural oscillator is generally characterized by a voltage-like state variable $V$. We assume that without arrival of fast inputs, $V$ is strictly increasing up to a spike threshold $\Theta_{V}>0$. When reaching the threshold at a time $t, V(t)=\Theta_{V}, V$ is reset to zero, i.e., $V\left(t^{+}\right)=0$, and starts increasing again. We denote the period of these free dynamics by $T$. We note that when $V(t)$ is specified by an autonomous differential equation (the function specifying the rate of change of $V$ does not depend on time) with unique solutions, trajectories cannot cross or overlap and furthermore the oscillatory behavior forbids fixed points. This implies strict monotonicity of $V$ except where $V$ is being reset.

We now introduce a so-called phase variable $\varphi(t)$, which increases with slope one in absence of fast input,

$$
\frac{d \varphi(t)}{d t}=1
$$

237 and has a phase threshold $\Theta$. When $\varphi$ reaches the threshold 238 at a time $t, \varphi(t)=\Theta$, the phase is reset to zero, $\varphi\left(t^{+}\right)=0$. 239 Note that Eq. (1) implies that the free period of the phase is $240 \Theta$. Since we want to map $\varphi(t)$ to $V(t)$, we choose the free periods identical, $\Theta=T$. The strict monotonicity of $V(t)$ then 241 implies that there is a strictly monotonic, bijective so-called rise 242 function $U$, mapping phase $\varphi$ to voltage $V$, i.e., at time $t$

$$
V(t)=U(\varphi(t)) .
$$

In particular, $\Theta_{V}$ and $\Theta$ are related by

$$
\Theta_{V}=U(\Theta) .
$$

For the LIF neuron, the type I neuron we focus on in our 245 study, $U:]-\infty, \Theta] \rightarrow]-\infty, \Theta_{V}$ ] (depending on the neuron 246 model domain and/or codomain are different). $U$ can be derived 247 directly from free membrane potential dynamics: Consider 248 free membrane potential dynamics $\widetilde{V}$, which start at the reset 249 potential at $t=0$, i.e., $\widetilde{V}(0)=0$. $\widetilde{V}$ can be continued for 250 negative times towards $-\infty$ (or a possible lower bound) and for 251 positive times to $\Theta_{V}$. The analogous dynamics of $\varphi$ run from 252 $-\infty$ (or a possible lower bound) to $\Theta=T$ with $\varphi(0)=0$. We ${ }_{253}$ have $U(\varphi)=\widetilde{V}(\varphi)$, since time equals phase for the considered 254 piece of dynamics.

When $\varphi$ reaches the phase threshold, it is reset and a spike is 256 emitted. After a delay time $\tau$, the spike arrives at postsynaptic 257 neurons at, say, time $t_{a}$. We assume that they respond with an 258 instantaneous jump in their membrane potential. The strength 259 $\varepsilon$ of the coupling from the pre- to the postsynaptic neuron 260 specifies the height of the jump. The corresponding phase jump 261 is computed using a transfer function $H$,

$$
\varphi\left(t_{a}^{+}\right)=H\left(\varphi\left(t_{a}\right), \varepsilon\right) .
$$

For convenience, we will omit $t_{a}$ and use $\varphi$ instead of $\varphi\left(t_{a}\right) .{ }_{263}$ If an input of strength $\varepsilon$ is subthreshold, i.e., $U(\varphi)+\varepsilon<\Theta_{V}, 264$ the transfer function is given by

$$
H(\varphi, \varepsilon)=U^{-1}(U(\varphi)+\varepsilon) .
$$

We may understand this formula as follows: We take $\varphi$ and 266 change to the membrane potential domain using $U$ given in 267 Eq. (2). We know that in the membrane potential domain an 268 input of strength $\varepsilon$ additively changes the membrane potential 269 $U(\varphi)$ by $\varepsilon$. We compute the corresponding phase, i.e., the 270 phase after the input, using $U^{-1}$. The composition of the steps, 271 $U^{-1}(U(\varphi)+\varepsilon)$, maps the phase before the interaction to the 272 phase after the interaction. We note that $H(\varphi, \varepsilon)$ is strictly ${ }_{273}$ monotonically increasing, both as a function of $\varepsilon$ and of $\varphi$, since 274 $U$ and thus $U^{-1}$ are strictly monotonically increasing. Since 275 suprathreshold input leads to immediate spiking and reset of 276 the neuron, we need to extend the definition of the transfer 277 function to

$$
\begin{gathered}
H(\varphi, \varepsilon)=U^{-1}(U(\varphi)+\varepsilon), \text { for } U(\varphi)+\varepsilon<\Theta_{V}, \\
H(\varphi, \varepsilon)=0, \text { for } U(\varphi)+\varepsilon \geqslant \Theta_{V} .
\end{gathered}
$$

$H(\varphi, \varepsilon)$ yields the new phase of a neuron when it receives an 279 input $\varepsilon$ at phase $\varphi$ [cf. Eq. (4)]. It is thus closely related to the 280 phase response curve (PRC) $P(\varphi, \varepsilon)$ (e.g., [3]), which yields 281 the phase change induced by an input $\varepsilon$ received at phase $\varphi, \quad{ }_{282}$

$$
P(\varphi, \varepsilon)=H(\varphi, \varepsilon)-\varphi .
$$

The infinitesimal phase response curve (iPRC) $Z(\varphi)$ char- 283 acterizes the phase shift of a neuron around $\varepsilon=0$; i.e., an 284 
(a)

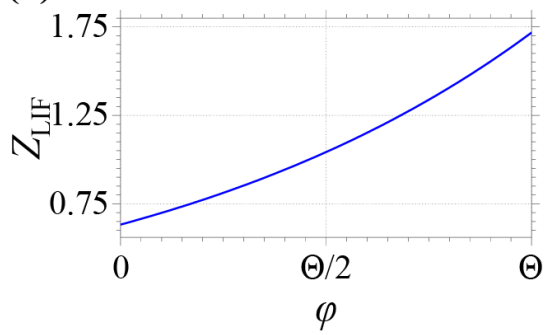

(d)

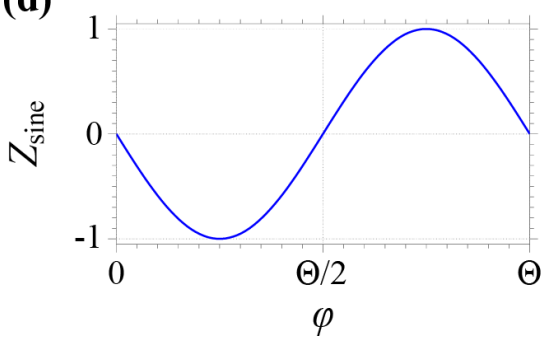

(b)

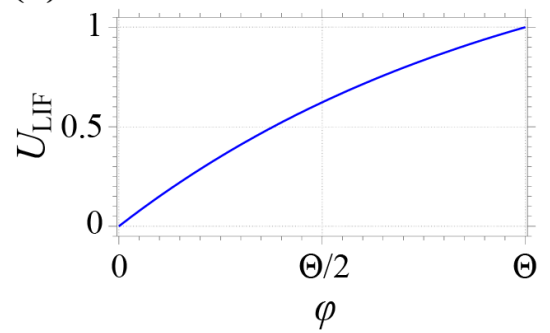

(e)

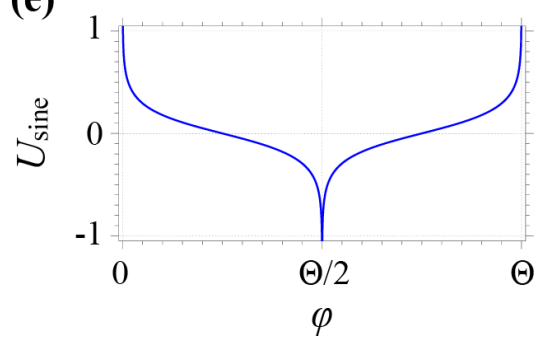

(c)

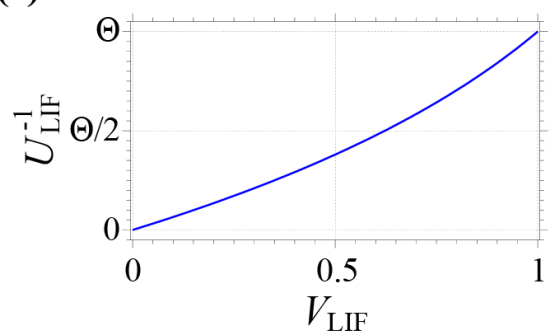

(f)

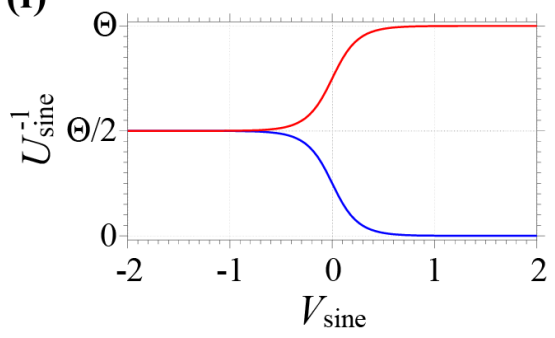

FIG. 1. Infinitesimal phase response curves (iPRC) $Z$, rise functions $(U)$, and inverse rise functions $\left(U^{-1}\right)$ for the type I leaky integrateand-fire neuron and the type II sine neuron. Upper panels show (a) the iPRC, (b) the rise function, and (c) the inverse rise function for the leaky integrate-and-fire neuron. Corresponding data are shown in the lower panels (d), (e), and (f) for the sine neuron; its inverse rise function has two branches (blue: $k=1$, red: $k=2$ ). Parameter setting: $\gamma=1, \Theta_{V}=1$, and $\Theta=1$.

infinitesimal input $d \varepsilon$ generates an infinitesimal phase shift

$$
d \varphi=Z(\varphi) d \varepsilon
$$

36 For small $\varepsilon$ around 0 we have $P(\varphi, \varepsilon) \approx Z(\varphi) \varepsilon$; 87 $H(\varphi, \varepsilon) \approx \varphi+Z(\varphi) \varepsilon . \quad Z(\varphi)$ and $H(\varphi, \varepsilon)$ are thus 88 related by

$$
Z(\varphi)=\left.\frac{\partial H(\varphi, \varepsilon)}{\partial \varepsilon}\right|_{\varepsilon=0}
$$

289 As mentioned above, $U^{-1}$ is strictly increasing. Equations (6) 290 and (8) then imply that $H$ and $P$ are strictly increasing in $\varepsilon$ for 291 subthreshold input. Because $P(\varphi, 0)$ equals $0, P(\varphi, \varepsilon)>0$ for $292 \varepsilon>0$ and subthreshold input. In other words, the PRC has to 293 be of type I; the formalism is thus applicable to type I neurons 294 only.

We now review the derivation of the phase representation for the type I LIF neuron using the methods described in Sec. II A (cf. also [65]). The dynamics of the membrane potential $V_{\mathrm{LIF}}(t)$ of the LIF neuron are given by

$$
\frac{d V_{\mathrm{LIF}}(t)}{d t}=-\gamma V_{\mathrm{LIF}}(t)+I,
$$

300 where $\gamma$ represents the inverse of the membrane time con301 stant and $I$ captures the external driving current. When the 302 membrane potential reaches its threshold $\Theta_{V}$, the neuron 303 spikes and the membrane potential is reset to zero. A spike arriving at time $t$ at a synaptic connection with strength $\varepsilon{ }_{304}$ induces an instantaneous change in the membrane potential, 305 i.e., $V_{\mathrm{LIF}}\left(t^{+}\right)=V_{\mathrm{LIF}}(t)+\varepsilon$. We assume that slow external 306 inputs add up to a constant current $I$, which drives the 307 neuron continuously over the threshold, such that it oscillates 308 "intrinsically" in absence of fast synaptic input. This allows us 309 to define the phase $-\infty<\varphi \leqslant \Theta$, which increases with slope 310 1 and is reset to zero when it reaches $\Theta$, where also a spike is 311 emitted.

The rise function $U$ linking the phase $\varphi$ of the spiking cycle ${ }_{313}$ to the membrane potential description $V$ can be determined as 314 described in Sec. II A as

$$
V_{\mathrm{LIF}}=U_{\mathrm{LIF}}(\varphi)=\frac{I}{\gamma}\left(1-e^{-\gamma \varphi}\right)
$$

(see $[4,65]$ ), yielding the inverse

$$
U_{\mathrm{LIF}}^{-1}\left(V_{\mathrm{LIF}}\right)=\frac{1}{\gamma} \ln \left(\frac{I}{I-\gamma V_{\mathrm{LIF}}}\right) .
$$

$U_{\mathrm{LIF}}$ is a monotonically increasing function of $\varphi$. Figures 1(b) 317 and 1 (c) show the rise function $U_{\mathrm{LIF}}$ and its inverse $U_{\mathrm{LIF}}^{-1}, 318$ respectively. The phase threshold is explicitly given in terms 319 of the voltage threshold $\Theta_{V}$ by

$$
\Theta=U_{\mathrm{LIF}}^{-1}\left(\Theta_{V}\right)=\frac{1}{\gamma} \ln \left(\frac{I}{I-\gamma \Theta_{V}}\right) .
$$

$U_{\mathrm{LIF}}$ and $U_{\mathrm{LIF}}^{-1}$ yield the transfer function of the LIF neuron ${ }_{321}$

$$
H_{\mathrm{LIF}}\left(\varphi, \varepsilon ; \Theta_{V}\right)= \begin{cases}-\frac{1}{\gamma} \ln \left(e^{-\gamma \varphi}-\frac{\gamma \varepsilon}{I}\right), & \text { for } U_{\mathrm{LIF}}(\varphi)+\varepsilon<\Theta_{V} \\ 0, & \text { for } U_{\mathrm{LIF}}(\varphi)+\varepsilon \geqslant \Theta_{V}\end{cases}
$$


(a)

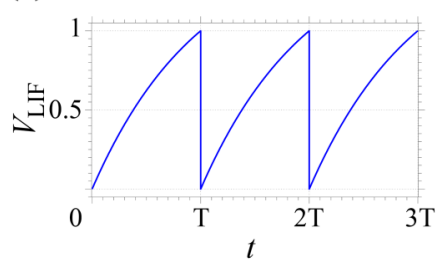

(d)

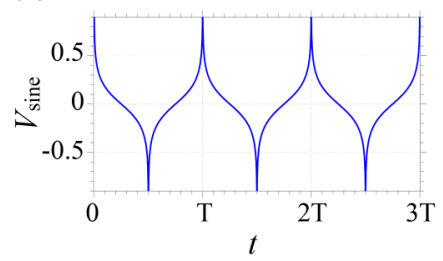

(b)

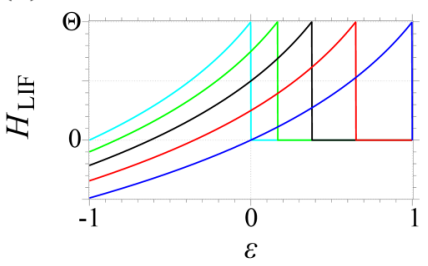

(e)

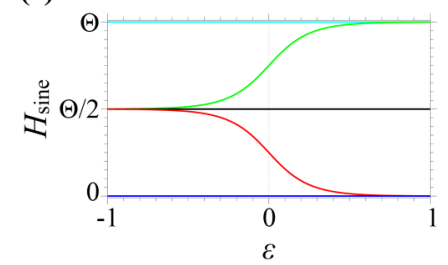

(c)

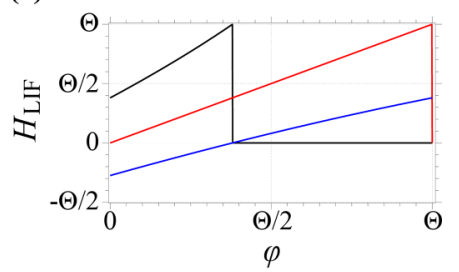

(f)

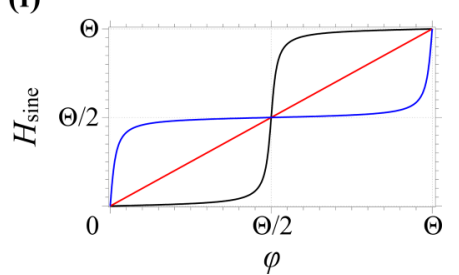

FIG. 2. Free dynamics $(V)$ and transfer functions $(H)$ for the type I leaky integrate-and-fire neuron and the type II sine neuron. Upper panels show (a) the free membrane potential dynamics, (b) the transfer function as a function of the coupling strength $\varepsilon$ for different constant values of the phase $\varphi$ at input arrival (blue, red, black, green, cyan: $\varphi=0,0.25,0.5,0.75$, and 1 ), and (c) the transfer function as a function of $\varphi$ for different constant $\varepsilon$ (blue, red, black: $\varepsilon=-0.5,0$, and 0.5 ) for the LIF neuron. Lower panels (d)-(f) show the corresponding plots for the sine neuron. Parameter setting: $\gamma=1, \Theta_{V}=1$, and $\Theta=1$.

22 cf. Eqs. (6) and (7). It is displayed in Fig. 2, panels (b) and (c).

Note that the phase $\varphi$ can assume all values within ] $-\infty, \Theta]$, where negative phases are generated by inhibitory inputs that cause hyperpolarization of the membrane potential.

Since we use the convention that the phase $\varphi$ is reset to zero ${ }^{33}$ when it reaches the threshold $\Theta$, at the time of a spiking
due to the driving current we have $\varphi=\Theta$ rather than $\varphi=0$.

Since $\gamma>0$, we can set $\gamma=1$ and $\Theta_{V}=1$ after appropriate scaling of time and voltage, without loss of generality for a single neuron. For simplicity, we assume that in networks with two type I neurons the membrane time constants are the same, such that the scaling is possible. The driving current $I$
335 that gives $U_{\mathrm{LIF}}(\Theta)=1$ follows in a straightforward way from 336 Eq. (12),

$$
I=\frac{1}{1-e^{-\Theta}} .
$$

The rise function Eq. (12) and its inverse Eq. (13) are then given by

$$
\begin{gathered}
U_{\mathrm{LIF}}(\varphi)=\frac{1-e^{-\varphi}}{1-e^{-\Theta}}, \\
U_{\mathrm{LIF}}^{-1}\left(V_{\mathrm{LIF}}\right)=-\ln \left[1-\left(1-e^{-\Theta}\right) V_{\mathrm{LIF}}\right] .
\end{gathered}
$$

339 Equations (15) and (16) yield the transfer function

$$
H_{\mathrm{LIF}}(\varphi, \varepsilon ; \Theta)= \begin{cases}-\ln \left[e^{-\varphi}-\left(1-e^{-\Theta}\right) \varepsilon\right], & \text { for } U_{\mathrm{LIF}}(\varphi)+\varepsilon<1, \\ 0, & \text { for } U_{\mathrm{LIF}}(\varphi)+\varepsilon \geqslant 1\end{cases}
$$

340 and, according to Eq. (10), the iPRC is given by

$$
Z_{\mathrm{LIF}}(\varphi ; \Theta)=\left(1-e^{-\Theta}\right) e^{\varphi},
$$

341 which is shown in Fig. 1(a).

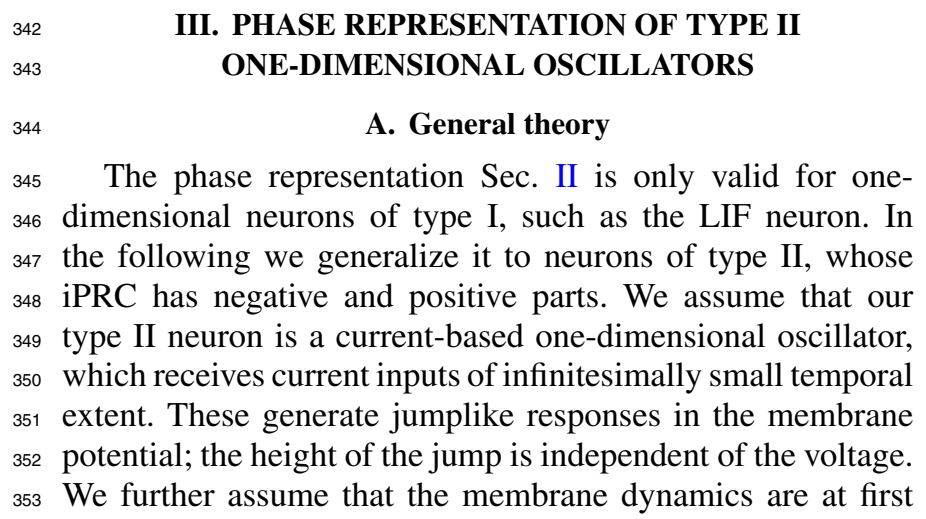

unknown, and the neuron dynamics are instead specified by an 354 infinitesimal phase response curve, which specifies the phase 355 response to input pulses of infinitesimally small strength. We ${ }_{356}$ then derive the free membrane dynamics as well as the full 357 phase representation. They turn out to follow nearly uniquely 358 from the iPRC for the considered class of oscillator models. $\quad 359$

The domain of the iPRC can be divided into several 360 intervals, in which the iPRC has the same sign (positive or ${ }_{361}$ negative). As an example, for a type I iPRC that is everywhere 362 larger than zero, we have only one interval $]-\infty, \Theta[$; cf. 363 the LIF neuron in Sec. IIB. For a sine-like type II iPRC, cf. 364 Sec. III B below, there are two subintervals $] 0, \Theta / 2[,] \Theta / 2, \Theta[, \quad 365$ and the iPRC becomes zero at the ends of the intervals. We aim 366 to construct rise functions for each subinterval and combine 367 them to obtain the transfer function $H$. 368

Restricted to a single interval $i$, the iPRC is either com- 369 pletely positive or negative. A strictly increasing free voltage 370 implies a positive iPRC: A small upward jump in the voltage 371 
372 maps the current state to a state that would be reached in 373 the future by free evolution; cf. Sec. II. A strictly decreasing 374 free voltage implies a negative iPRC, as an upward jump in 375 the voltage maps the current state to an earlier state. In turn, 376 a positive (negative) iPRC implies monotonically increasing 377 (decreasing) free voltage dynamics. We note that this implies 378 that a differential equation specifying $V$ must switch between 379 intervals with different signs of the iPRC (cf. Sec. III B below). 380 In interval $i$ we can define a monotonically increasing or 381 decreasing transfer function $U_{i}$, which maps phase to voltage, 382 cf. Eq. (2), as follows: For given $\varphi$, there are sufficiently small ${ }_{383}$ inputs $\varepsilon$ such that the voltage and phase stay within the interval 384 even if $i$ is the interval neighboring the threshold. Then, the 385 transfer function is given by Eq. (5) and

$$
\frac{\partial H_{i}(\varphi, \varepsilon)}{\partial \varepsilon}=\frac{1}{U_{i}^{\prime}\left(U_{i}^{-1}\left(U_{i}(\varphi)+\varepsilon\right)\right)} .
$$

386 By setting $\varepsilon$ to 0 , see Eq. (10), we obtain for all $\varphi$ in the interval

$$
Z(\varphi)=\left.\frac{\partial H_{i}(\varphi, \varepsilon)}{\partial \varepsilon}\right|_{\varepsilon=0}=\frac{1}{U_{i}^{\prime}\left(U_{i}^{-1}\left(U_{i}(\varphi)\right)\right)}=\frac{1}{U_{i}^{\prime}(\varphi)} .
$$

387 The slope of $U_{i}(\varphi)$ specifies $U_{i}(\varphi)$ up to a constant, so ${ }_{388} U_{i}(\varphi)$ is basically the antiderivative $F_{i}(\varphi)$ of $1 / Z(\varphi)$ in 389 interval $i$,

$$
F_{i}(\varphi)=\int \frac{1}{Z(\varphi)} d \varphi .
$$

390 We obtain $U_{i}(\varphi)$ from $F_{i}(\varphi)$ by specifying the voltage at some 391 phase.

392 When $\varphi$ approaches an interval boundary where the iPRC 393 has a zero, $U_{i}(\varphi)$ and thus the voltage will usually tend to $\pm \infty$, 394 which we then take as the value assumed by the rise function 395 there. We note that the voltage can tend to $+\infty$ even if the 396 phase is not in the interval neighboring the threshold. Then 397 the phase does not reach the phase threshold and the neuron 398 does not spike. Models with this property may be interpreted as 399 having a history-dependent voltage spike threshold. We note 400 that our formalism allows us to construct oscillator models 401 from the iPRC for which $U_{i}(\varphi)$ does not have a reasonable 402 biological interpretation in terms of a voltage. As an example, 403 an iPRC that is negative in the interval adjacent to the phase 404 threshold can give rise to a $U_{i}(\varphi)$ that reaches $-\infty$ as the phase 405 approaches the phase threshold and the neuron spikes.

406 If $\varepsilon$ does not lead the dynamics out of interval $i$, the transfer 407 function is given by

$$
H_{i}(\varphi, \varepsilon)=U_{i}^{-1}\left(U_{i}(\varphi)+\varepsilon\right) .
$$

408 It is uniquely determined by the iPRC, since adding a constant 409 to $U_{i}$, i.e., using $U_{i, c_{i}}(\varphi)=U_{i}(\varphi)+c_{i}$ to define $H_{i}$, does not 410 change it,

$$
\begin{aligned}
H_{i}(\varphi, \varepsilon) & =U_{i, c_{i}}^{-1}\left(U_{i, c_{i}}(\varphi)+\varepsilon\right)=U_{i}^{-1}\left(U_{i}(\varphi)+c_{i}+\varepsilon-c_{i}\right) \\
& =U_{i}^{-1}\left(U_{i}(\varphi)+\varepsilon\right) .
\end{aligned}
$$

411 We can derive the rise function also in a more intuitive 412 manner as follows: An input to our neuron models should have ${ }_{413}$ the same effect whether we apply it at once or in small pieces, 414 which we may imagine to be separated by small temporal differences. Indeed, in the membrane potential representation, 415 the input is simply additive, so this is certainly satisfied. In 416 phase representation, it should be satisfied as well. An input ${ }_{417}$ $d \tilde{\varepsilon}$ arriving at phase $\varphi$ leads in linear approximation to a new ${ }_{418}$ phase $\varphi^{+}=\varphi+Z(\varphi) d \tilde{\varepsilon}$. If the change due to an input piece ${ }_{419}$ $d \tilde{\varepsilon}$ does not depend on the total input $\varepsilon$, we should get the 420 same change, if the previous phase has been reached due to ${ }_{421}$ a previous piece $\tilde{\varepsilon}$ of an input. Denoting the phase before the ${ }_{422}$ arrival of $d \tilde{\varepsilon}$ by $\varphi(\tilde{\varepsilon})$, we find that the input $\tilde{\varepsilon}+d \tilde{\varepsilon}$ leads to ${ }_{423}$ the phase $\varphi(\tilde{\varepsilon}+d \tilde{\varepsilon})=\varphi(\tilde{\varepsilon})+Z(\varphi(\tilde{\varepsilon})) d \tilde{\varepsilon}$. Note that $\varphi(\tilde{\varepsilon})$ is 424 the exact nonapproximated phase after receiving $\tilde{\varepsilon}$, while the 425 impact of $d \tilde{\varepsilon}$ is covered up to first order. Knowing the impact ${ }_{426}$ of an additional input $d \tilde{\varepsilon}$ up to first order (equivalently, the ${ }_{427}$ impact of an infinitesimal input) allows us to write the phase 428 change in the form of a differential equation,

$$
\frac{d \varphi(\tilde{\varepsilon})}{d \tilde{\varepsilon}}=Z(\varphi(\tilde{\varepsilon}))
$$

Since the impact of an input piece does not explicitly depend 430 on the previously received input, the right-hand side does not ${ }_{431}$ explicitly depend on the independent variable $\tilde{\varepsilon}$, but only via ${ }_{432}$ $\varphi(\tilde{\varepsilon})$. In other words, the phase change $\varphi(\tilde{\varepsilon})$ is characterized by ${ }_{433}$ an autonomous ordinary differential equation. In the Appendix, 434 we highlight that general phase oscillators do not have this 435 property, using the radial isochron clock. Note that Eq. (28) 436 can also be derived by discretizing the timelike variable $\varepsilon$ into ${ }_{437}$ many small steps of size $d \tilde{\varepsilon}$, expanding the PRC around zero ${ }_{438}$ coupling strength by its Taylor series, and taking the limit of 439 $d \tilde{\varepsilon} \rightarrow 0$.

Solving Eq. (28) by separation of variables, we obtain

$$
\int_{\varphi}^{\varphi^{+}} \frac{1}{Z(\varphi)} d \varphi=\int_{0}^{\varepsilon} d \tilde{\varepsilon}=\varepsilon
$$

where $\varphi^{+}$and $\varphi$ are the phases before and after arrival of the ${ }_{442}$ total subthreshold input $\varepsilon$. By the first fundamental theorem of ${ }_{443}$ calculus, we have $F_{i}\left(\varphi^{+}\right)-F_{i}(\varphi)=\varepsilon$, where again $F_{i}(\varphi)={ }_{444}$ $\int 1 / Z(\varphi) d \varphi$. Since on the other hand

$$
U_{i}\left(\varphi^{+}\right)-U_{i}(\varphi)=\varepsilon,
$$

$F_{i}$ equals $U_{i}$ up to an additive constant and $U_{i}$ is basically the ${ }_{446}$ antiderivative of $1 / Z(\varphi)$ in the interval $i$.

Equation (28) and its property of being autonomous can also 448 be directly derived from the fact that $d V_{i}$ (the change of the ${ }_{449}$ voltage due to $d \tilde{\varepsilon}$ ) does not explicitly (not even implicitly) de- 450 pend on already applied subthreshold input: While receiving an ${ }_{451}$ input, $V_{i}$ may be seen as a function $V_{i}(\tilde{\varepsilon})$ of the already applied ${ }_{452}$ piece of input $\tilde{\varepsilon}$, with initial value $V_{i}(0)=V_{i}$ and $\tilde{\varepsilon}$ running ${ }_{453}$ from 0 to $\varepsilon . V_{i}(\tilde{\varepsilon})$ then satisfies the autonomous differential ${ }_{454}$ equation $d V_{i}(\tilde{\varepsilon}) / d \tilde{\varepsilon}=1$. This implies $d U_{i}(\varphi(\tilde{\varepsilon})) / d \tilde{\varepsilon}=1$ and, 455 after application of the chain rule, the differential equation 456 $d \varphi(\tilde{\varepsilon}) / d \tilde{\varepsilon}=1 / U_{i}^{\prime}(\varphi(\tilde{\varepsilon}))$. Since for $\tilde{\varepsilon}=0$ the left-hand side ${ }_{457}$ equals $Z(\varphi)$ and the differential equation is autonomous, we 458 have $1 / U_{i}^{\prime}(\varphi)=Z(\varphi)$ for all phases. This implies that $\varphi(\tilde{\varepsilon}){ }_{459}$ satisfies Eq. (28) and it implies Eq. (30).

Equation (28) also allows us to directly derive the transfer ${ }_{461}$ function and thus the complete phase representation from the 462 iPRC. We note that $\varphi(\tilde{\varepsilon})=H_{i}(\varphi, \tilde{\varepsilon})$ and rewrite Eq. (28) as ${ }_{463}$

$$
\frac{\partial H_{i}(\varphi, \tilde{\varepsilon})}{\partial \tilde{\varepsilon}}=Z\left(H_{i}(\varphi, \tilde{\varepsilon})\right)
$$


464 with initial condition $H_{i}(\varphi, 0)=\varphi$, which reduces to Eq. (10) 465 for $\tilde{\varepsilon}=0$. Solving the differential equation yields the transfer 466 function in interval $i$.

${ }_{467}$ Phases $\varphi$ where the iPRC is zero are fixed points of the 468 dynamics Eqs. (28) and (31). Thus, under weak conditions 469 on Eq. (28) (the iPRC is globally Lipschitz continuous such 470 that the differential equation has a unique solution existing 471 for all $\varepsilon$ ), such a $\varphi$ will not be changed by input, $H_{i}(\varphi, \varepsilon)=$ ${ }_{472} \varphi=$ constant; furthermore, no finite input will lead beyond the 473 borders of an interval $i$ where the iPRC gets zero.

\section{$474 \quad$ B. The sine neuron in phase representation}

475 Typical type II neurons show a phase delay in response 476 to excitatory input $\varepsilon>0$ arriving at small phases (early in 477 the spiking cycle, shortly after a spike) and a phase advance 478 when such input arrives at larger phases [3,57]. With these 479 characteristics in mind, we define our type II neurons as "sine 480 neurons" by an iPRC,

$$
Z_{\text {sine }}(\varphi)=-\sin \left(\frac{2 \pi}{\Theta} \varphi\right),
$$

481 where $\varphi \in[0, \Theta]$ [see Fig. $1(\mathrm{~d})]$ and $\Theta \equiv T$ is the period 482 and the phase threshold of the neuron. We use the sinusoidal 483 function as the iPRC of our type II neurons also because neuron 484 models such as the Hodgkin-Huxley neuron can undergo Hopf 485 bifurcations $[66,67]$ and the normal form oscillator of Hopf 486 bifurcating systems and thus general Hopf bifurcating systems 487 with appropriate parameters have near the bifurcation for 488 suitable inputs a sinusoidal iPRC Eq. (32) [68]. To facilitate 489 the analytical study of two-neuron networks that include type 490 II neurons, we apply the phase oscillator formalism to the sine 491 neuron. Since the iPRC changes sign, we use the methodology 492 derived in Sec. III A.

${ }_{493}$ We split the interval domain $[0, \Theta]$ of $Z_{\text {sine }}$ into two, i.e., $\left.{ }_{494}\right] 0, \Theta / 2[$ and $] \Theta / 2, \Theta\left[\right.$, and treat $U_{\text {sine }}(\varphi)$ at $\varphi \in\{0, \Theta / 2, \Theta\}$ 495 separately. Equations (25) and (32) yield the rise functions 496 for the first subinterval $\left(U_{\text {sine }, 1}(\varphi), \varphi \in\right] 0, \Theta / 2[)$ and for the 497 second subinterval $\left(U_{\text {sine }, 2}(\varphi), \varphi \in\right] \Theta / 2, \Theta[): U_{\text {sine }, \mathrm{k}}(\varphi)=$ ${ }_{498}-\Theta \ln [|\tan (\pi \varphi / \Theta)|] / 2 \pi+c_{k}$, where $c_{k} \in \mathbb{R}$ and $k \in\{1,2\}$. 499 From the first subinterval, we compute the values of 500 the rise function at $\varphi=0$ and $\varphi=\Theta / 2, \quad U_{\text {sine }}(0)=$ $501 \lim _{\varphi \rightarrow 0^{+}} U_{\text {sine, } 1}(\varphi)=\infty, U_{\text {sine }}(\Theta / 2)=\lim _{\varphi \rightarrow \Theta^{-} / 2} U_{\text {sine }, 1}(\varphi)=$ $502-\infty$. Compatible with this, $\lim _{\varphi \rightarrow \Theta^{+} / 2} U_{\text {sine, } 2}(\varphi)=-\infty$. 503 Finally, at $\varphi=\Theta, U_{\text {sine }}(\Theta)=\lim _{\varphi \rightarrow \Theta^{-}} U_{\text {sine }, 2}(\varphi)=\infty$. In 504 summary, the rise function of the sine neuron is given by

$$
U_{\text {sine }}(\varphi)= \begin{cases}\infty, & \text { for } \varphi \in\{0, \Theta\}, \\ -\infty, & \text { for } \varphi=\Theta / 2, \\ -\frac{\Theta}{2 \pi} \ln \left[\tan \left(\frac{\pi}{\Theta} \varphi\right)\right]+c_{1}, & \text { for } \varphi \in] 0, \frac{\Theta}{2}[, \\ -\frac{\Theta}{2 \pi} \ln \left[\tan \left(-\frac{\pi}{\Theta} \varphi\right)\right]+c_{2}, & \text { for } \varphi \in] \frac{\Theta}{2}, \Theta[.\end{cases}
$$

505 Figure 1(e) illustrates the rise function $U_{\text {sine }}(\varphi)$ for the sine 506 neuron with $c_{1}=c_{2}=0$.

507 Since the membrane potential of our sine neuron satis${ }_{508}$ fies $V_{\text {sine }}(t)=U_{\text {sine }}(\varphi(t))$, it reaches $+\infty$ in finite time [see 509 Fig. 1(e)]. We can thus set the spike threshold to $\infty$. In 510 this respect, the sine neuron resembles the theta or quadratic

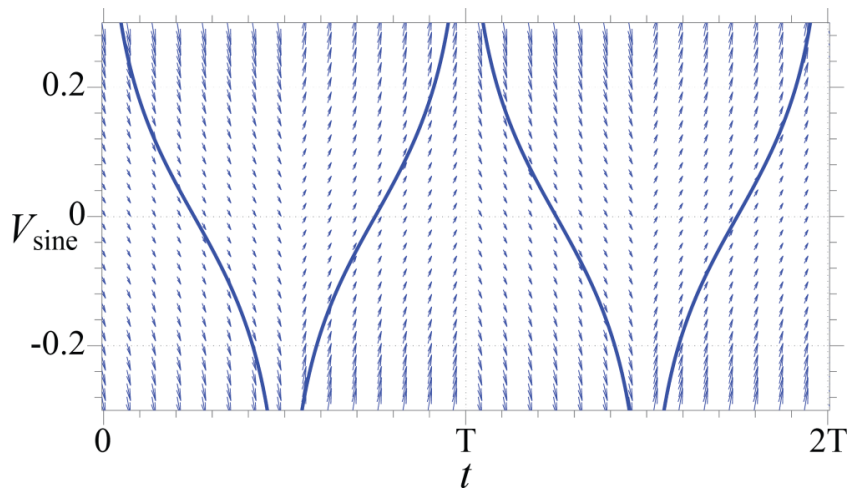

FIG. 3. Vector field of the sine neuron defined by Eqs. (34) and (35). The solid curves represent $V_{\text {sine }}(t)=U_{\text {sine }}(\varphi(t))$ for $c_{1}=c_{2}=$ 0 . The vector field switches when $V_{\text {sine }}$ reaches $+\infty$ or $-\infty$.

integrate-and-fire model (see, e.g., [1] and Sec. VII). How- 511 ever, the sine neuron is not reset to $-\infty$. When it reaches 512 threshold, the membrane potential decreases from $+\infty$ to $-\infty \quad \infty 13$ halfway through the cycle by its intrinsic dynamics. In this 514 regime, excitatory input yields a phase delay. Thereafter the 515 membrane potential increases gradually to $+\infty$ in a regime 516 where excitation yields a phase advance. The dynamical 517 regime thus depends on the last "event." If the last event was 518 sending a spike $\left(V_{\text {sine }}=\infty\right)$, we are in regime $k=1$, where 519 excitation delays the phase. If the last event was reaching the 520 reset potential $\left(V_{\text {sine }}=-\infty\right)$, we are in regime $k=2$, where ${ }_{521}$ excitation advances the phase. Note that this is an extension 522 to the dynamics of standard integrate-and-fire models, where 523 neurons are only in one dynamical regime and reset in an 524 infinitesimally short time after they reach threshold. In contrast 525 to the "spike response" extension (see [69]), the dynamical 526 regime in our extension does not only depend on the time ${ }_{527}$ elapsed since spike sending, but also on the full dynamics of the 528 neuron. A stronger asymmetry between spiking and reset or a 529 more rapid onset of spikes can be easily achieved by modifying 530 the sinusoidal shape of the iPRC.

Interestingly, the membrane potential of our sine neuron obeys the simple nonlinear differential equation

$$
\frac{d V_{\text {sine }}(t)}{d t}=\frac{d U_{\text {sine }}(\varphi)}{d \varphi} \frac{d \varphi(t)}{d t}=-\cosh \left[\frac{2 \pi}{\Theta} V_{\text {sine }}(t)\right]
$$

in the regime $k=1$, i.e., if the previous event was a spike, and 534 it obeys

$$
\frac{d V_{\text {sine }}(t)}{d t}=\cosh \left[\frac{2 \pi}{\Theta} V_{\text {sine }}(t)\right]
$$

in the regime $k=2$, i.e., if the previous event was a reset; cf. ${ }_{536}$ Fig. 3.

Using Eq. (33), we can define an inverse function $U_{\text {sine }}^{-1}$ with ${ }_{538}$ two branches; see Fig. 1(f). For the branch $k=1$ the inverse 539 function $U_{\text {sine }}^{-1}$ maps the state variable $V_{\text {sine }} \in$ ] $-\infty, \infty$ [ to the 540 phase $\varphi \in] 0, \Theta / 2[$ by 541

$$
U_{\text {sine }}^{-1}\left(V_{\text {sine }}\right)=\frac{\Theta}{\pi} \arctan \left(e^{-\frac{2 \pi}{\Theta}\left(V_{\text {sine }}-c_{1}\right)}\right) .
$$


542 For the branch $k=2$, the inverse function $U_{\text {sine }}^{-1}$ maps the 543 membrane potential $V_{\text {sine }}$ in the range $]-\infty, \infty[$ to $] \Theta / 2, \Theta[$,

$$
U_{\text {sine }}^{-1}\left(V_{\text {sine }}\right)=-\frac{\Theta}{\pi} \arctan \left(e^{-\frac{2 \pi}{\Theta}\left(V_{\text {sine }}-c_{2}\right)}\right)+\Theta .
$$

44 Using these branches, we can now construct the transfer 545 function $H_{\text {sine }}(\varphi)$. For this, we first consider the membrane 546 potential dynamics and note that an input $\varepsilon$ cannot bring $V_{\text {sine }}$ 547 above $+\infty$ or below $-\infty$. As a consequence, inputs do not 58 alter the dynamical regime $k$. To compute the phase after an 54 input we therefore have to use Eq. (36) if the original phase $\varphi$ is 50 within $] 0, \Theta / 2[$ (regime $k=1$ ) and Eq. (37) if $\varphi \in] \Theta / 2, \Theta[$ 551 (regime $k=2$ ). Further taking into account that the transfer 552 function is the identity for any input at $\varphi \in\{0, \Theta / 2, \Theta\}$ (the 553 zeros of the PRC; see Sec. III A), we arrive at $H_{\text {sine }}(\varphi, \varepsilon)$ :

$$
\begin{aligned}
& H_{\text {sine }}(\varphi, \varepsilon) \\
& = \begin{cases}U_{\text {sine }, 1}^{-1}\left(U_{\text {sine }}(\varphi)+\varepsilon\right), & \text { for } \varphi \in] 0, \frac{\Theta}{2}[, \\
U_{\text {sine }, 2}^{-1}\left(U_{\text {sine }}(\varphi)+\varepsilon\right), & \text { for } \varphi \in] \frac{\Theta}{2}, \Theta[, \\
\varphi, & \text { for } \varphi \in\left\{0, \frac{\Theta}{2}, \Theta\right\},\end{cases} \\
& = \begin{cases}\frac{\Theta}{\pi} \arctan \left[\tan \left(\frac{\pi}{\Theta} \varphi\right) e^{-\frac{2 \pi \varepsilon}{\Theta}}\right], & \text { for } \varphi \in] 0, \frac{\Theta}{2}[, \\
\frac{\Theta}{\pi} \arctan \left[\tan \left(\frac{\pi}{\Theta} \varphi\right) e^{-\frac{2 \pi \varepsilon}{\Theta}}\right]+\Theta, & \text { for } \varphi \in] \frac{\Theta}{2}, \Theta[, \\
\varphi, & \text { for } \varphi \in\left\{0, \frac{\Theta}{2}, \Theta\right\} .\end{cases}
\end{aligned}
$$

554 Figures 2(e) and 2(f) show the transfer function as a function of 555 synaptic increment $\varepsilon$ and as a function of phase $\varphi$, respectively. 556 The panels illustrate, in particular, that $\varphi$ can assume values in $557[0, \Theta]$, that the neuron cannot be excited suprathresholdly, and 558 that inputs do not give rise to transitions between the regimes ${ }_{559} k=1$ and $k=2$. We note that in phase representation, we do 560 not have to keep track of the type of the last event to execute 561 the dynamical evolution since this information is contained in 562 the current phase.

\section{3 564 \\ IV. INTERACTION SCENARIOS, ITERATION MAP, AND PHASE-LOCKING EQUATIONS}

\section{A. Interaction scenarios}

566 567 an excitatory (henceforth E) and an inhibitory (henceforth 568 I) neuron [cf. Fig. 4(a)]. They represent two synchronized 569 coupled neuron populations, an excitatory and an inhibitory 570 population, by one representative neuron for each population. 571 The couplings between the neuron populations are accounted 572 for by couplings between the two representative neurons. We 573 aim at setting up an event-based iteration map in the phase 574 variables, which fully describes the network dynamics. Its fixed 575 points and periodic orbits correspond to periodic oscillations 576 in the phase dynamics (cf., e.g., [70]). To derive the map, we 577 consider the difference of shifted phases of the two neurons 578 and describe how it changes when the neurons send and receive 579 spikes. We focus on regular periodic oscillations, where the $\mathrm{E}$ 580 and I neurons spike once per cycle, argue which fixed points or 581 periodic orbits in the dynamics correspond to ING and PING 582 rhythms, and explore when they are generated and how they 583 give way to each other.
We incorporate couplings from $E$ to I (strength $\varepsilon_{E \rightarrow I}$ ), from 584 I to $\mathrm{E}\left(\varepsilon_{I \rightarrow E}\right)$, and self-inhibition from I to itself $\left(\varepsilon_{I \rightarrow I}\right)$. For 585 simplicity, we do not consider self-excitation from E to itself, 586 as it is not critically involved in PING or ING rhythms. Five 587 events can take place in such networks: spiking of the E neuron, ${ }_{588}$ spiking of the I neuron, arrival of a spike from the E neuron ( ${ }_{589}$ spike) at the I neuron, arrival of a spike from the I neuron (I 590 spike) at the E neuron, and arrival of an I spike at the I neuron. 591 When an event occurs, the phase difference between the E and 592 I neurons typically changes. We choose the conduction delay 593 between spike sending and receiving to be $\tau$ for all connections 594 to reduce the number of free parameters. Further, we assume 595 that the neurons do not oscillate with too high frequencies 596 (intrinsic period is longer than $2 \tau$ ) to ensure that a spike does 597 not arrive in the next cycle. Finally, we assume that inhibition 598 always induces a phase delay in the E neuron. Due to the 599 finite delay $\tau$, spikes of the two neurons can overlap in the 600 sense that one neuron spikes, while a spike sent by the other 601 neuron has not yet arrived. To deal with this, we construct 602 nonoverlapping interaction scenarios, each containing a series 603 of events. Each of the scenarios defines a local iteration map. 604 The local maps can be combined to a global one, $G$, which acts 605 on a single variable $\Delta \psi$, the difference of shifted phases of the 606 two neurons taking into account the differences in intrinsic 607 period.

Without any restriction on firing activities of the E and 609 I neurons, the events can be combinatorially combined in 610 infinitely many ways, which results in infinitely many in- 611 teraction scenarios. However, under the assumptions made 612 in the previous paragraphs, there are five oscillation-relevant ${ }_{613}$ interaction scenarios; cf. the five panels in Fig. 4(b). Each 614 interaction scenario gives rise to a local iteration map, which 615 maps the difference of shifted phases $\Delta \psi$ before the scenario $\quad 616$ to the difference of shifted phases $\Delta \widetilde{\psi}$ after the scenario. In 617 scenario 1, the I neuron spikes and the spike is received before 618 any other event, in particular, before the E neuron spikes. 619 Similarly, in scenario 5 the E neuron spikes and the spike 620 is received before any other event, in particular, before the I 621 neuron spikes. In regular rhythms, scenario 1 must be followed 622 by scenario 5 and vice versa. However, in general periodic 623 oscillations, scenario 1 is not necessarily tied to scenario 5624 and we therefore do not combine them into one scenario. 625 We note that if scenario 1 follows shortly after scenario 5, 626 the corresponding rhythm is PING, since the E input nearly ${ }_{627}$ generates the spiking of the I neuron (see Sec. VII for further ${ }_{628}$ discussion). If the time difference is larger, the character of the ${ }_{629}$ rhythm becomes unclear. However, for the considered sets of 630 parameters around the crossing of pure ING and pure PING 631 network oscillation frequencies, we find in our simulations that 632 scenario 1 always follows shortly after scenario 5 in regular 633 oscillations (less than $0.1 T$, where $T$ is the network oscillation 634 period). For simplicity, we thus denote every scenarios 5,1 in 635 alternation rhythm as PING in the following. We note that ${ }_{636}$ scenario 1 will usually not shortly precede scenario 5 , since ${ }_{637}$ the I-spike arrival at the end of scenario 1 has a retarding effect 638 on E-spike generation, which starts scenario 5. In scenario 2 the 639 I neuron spikes, followed by the E neuron before the inhibitory 640 input from the I neuron arrives and can hinder it. Since the I 641 neuron spikes due to its own drive while the input from the 642 E neuron arrives shortly thereafter, this scenario gives rise to 643 
(a)

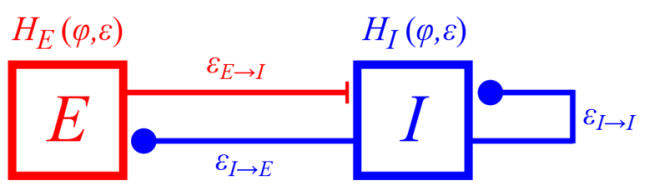

(b)
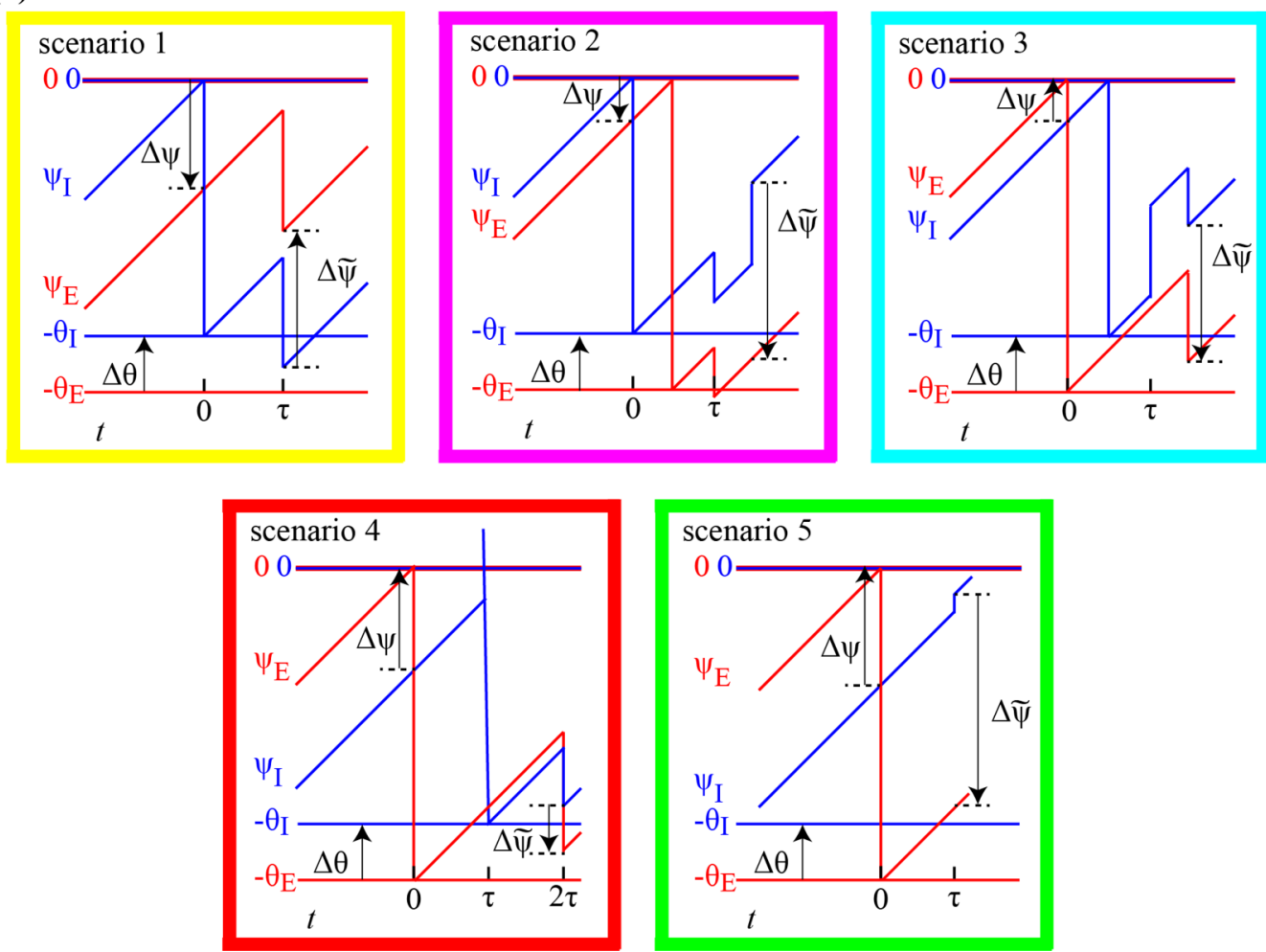

FIG. 4. Network of two neurons and illustrations of the five possible scenarios for interactions between them. Panel (a) displays the neurons (E: an excitatory neuron, I: an inhibitory neuron) and the couplings between them; their responses to inputs are governed by $H_{E}(\varphi, \varepsilon)$ and $H_{I}(\varphi, \varepsilon)$, respectively. Panels (b) show the dynamics of the shifted phases $\psi_{E}$ (red) and $\psi_{I}$ (blue) in scenarios $1-5$. The scenarios are arranged according to the initial value of the phase difference $\Delta \psi$ [Eq. (42)], starting from large magnitude negative values.

644 an ING rhythm. In scenario 3, the E neuron spikes, followed 645 by the I neuron, which spikes before the input from the $\mathrm{E}$ 646 neuron arrives. Although the sequence of spiking of the $\mathrm{E}$ and 647 I neurons is reminiscent of PING, this scenario also gives rise 648 to an ING rhythm, since the I neuron does not spike due to 649 excitatory input from the $\mathrm{E}$ neuron, but again due to its own 650 drive. In scenario 4, again first the E neuron spikes, followed 651 by the I neuron. However, the I neuron now spikes due to the ${ }_{652}$ excitatory input from the E neuron, which lets the I neuron ${ }_{653}$ exceed the spike threshold. This scenario is thus typical for 654 PING.

\section{B. Phase dynamics}
We will now consider the interaction scenarios and their 657 impact on the phases in detail. To identify quantities related to 658 the $\mathrm{E}$ and $\mathrm{I}$ neurons, we endow them with an index $E$ and $I$ : In 659 particular, $\varphi_{E}\left(\varphi_{I}\right)$ and $\Theta_{E}\left(\Theta_{I}\right)$ are phase and phase threshold 660 of the $\mathrm{E}(\mathrm{I})$ neuron. To study neurons with different intrinsic ${ }_{661}$ periods $\left(\Theta_{E} \neq \Theta_{I}\right)$, we introduce new, shifted phase variables $662 \psi_{E}$ and $\psi_{I}$, which describe the remaining phases of the $\mathrm{E}$ and

I neurons to the threshold,

$$
\begin{gathered}
\psi_{E}=\varphi_{E}-\Theta_{E}, \\
\psi_{I}=\varphi_{I}-\Theta_{I} .
\end{gathered}
$$

The neurons spike at $\psi_{E}=0$ and $\psi_{I}=0$, and the shifted ${ }_{664}$ phases are thereafter reset to $-\Theta_{E}$ and $-\Theta_{I}$. The remain- 665 ing times to the next spiking generated by purely intrinsic 666 dynamics are given by $-\psi_{E} \geqslant 0$ and $-\psi_{I} \geqslant 0$. We denote ${ }_{667}$ the differences between the new, shifted phases, the standard 668 phases, and the phase thresholds (periods) of the neurons by $\quad{ }_{669}$

$$
\begin{aligned}
\Delta \psi & =\psi_{E}-\psi_{I}, \\
\Delta \varphi & =\varphi_{E}-\varphi_{I}, \\
\Delta \Theta & =\Theta_{E}-\Theta_{I},
\end{aligned}
$$

respectively. Equations (40) and (41) yield the relation

$$
\Delta \psi=\Delta \varphi-\Delta \Theta .
$$

We will now derive the transition from $\Delta \psi$ before to $\Delta \widetilde{\psi} \quad 671$ after the sequence of interactions for scenarios $1-5$ and for 672 
673 a scenarios 5,1 pair. Without loss of generality, we assume $674 t=0$ at the start of each scenario.

\section{5 \\ C. Scenario 1}

676

Scenario 1, where only the I neuron spikes, occurs for

$$
\Delta \psi \leqslant-\tau
$$

677 The phase $\psi_{I}$ of the I neuron (henceforth "I phase") and the 678 phase $\psi_{E}$ of the E neuron (henceforth "E phase") at the start 679 of the interaction sequence at $t=0$ are

$$
\begin{gathered}
\psi_{I}=0, \\
\psi_{E}=\Delta \psi .
\end{gathered}
$$

680 The interaction sequence in scenario 1 consists of sending and 681 receiving an I spike. The I neuron is reset after spiking. Thus, 682 it receives its own spike while having the phases [cf. Eq. (1)]

$$
\begin{gathered}
\varphi_{I}(\tau)=\tau, \\
\psi_{I}(\tau)=\varphi_{I}(\tau)-\Theta_{I}=\tau-\Theta_{I} .
\end{gathered}
$$

683 After input processing and thus directly at the end of the 684 interaction sequence, the phases are

$$
\begin{gathered}
\widetilde{\varphi}_{I}=H_{I}\left(\tau, \varepsilon_{I \rightarrow I}\right), \\
\widetilde{\psi}_{I}=H_{I}\left(\tau, \varepsilon_{I \rightarrow I}\right)-\Theta_{I} .
\end{gathered}
$$

685 The E neuron receives the I spike while having a phase $\varphi_{E}(0)+$ ${ }_{686} \tau=\Theta_{E}+\Delta \psi+\tau$. The phases of the E neuron directly after 687 the interaction sequence are thus

$$
\begin{gathered}
\widetilde{\varphi}_{E}=H_{E}\left(\Theta_{E}+\Delta \psi+\tau, \varepsilon_{I \rightarrow E}\right) \\
\widetilde{\psi}_{E}=\widetilde{\varphi}_{E}-\Theta_{E}=H_{E}\left(\Theta_{E}+\Delta \psi+\tau, \varepsilon_{I \rightarrow E}\right)-\Theta_{E} .
\end{gathered}
$$

688 Equations (54) and (52) yield the phase difference after the 689 interaction,

$$
\Delta \widetilde{\psi}=\underbrace{H_{E}\left(\Theta_{E}+\Delta \psi+\tau, \varepsilon_{I \rightarrow E}\right)-H_{I}\left(\tau, \varepsilon_{I \rightarrow I}\right)-\Delta \Theta}_{=: G(\Delta \psi)} .
$$

${ }_{690} G$ maps the difference of the shifted phases before the interac691 tion sequence to the difference of the shifted phases thereafter. 692 Scenario 1 can only generate a regular oscillation (syn693 chronization between neurons of order $1: 1$ [71]) together 694 with scenario 5 (see the related paragraph below). However, 695 scenario 1 can repeat to give rise to a regular oscillation of 696 the I neuron, where the E neuron is suppressed. For such an 697 oscillation, $\Delta \psi$ is given by the solution of

$$
G(\Delta \psi)=\Delta \psi .
$$

${ }_{698}$ This is because $\Delta \psi$ does not change between scenarios and 699 there is only one scenario repeating, so $\Delta \psi$ at its beginning and 700 ending must be the same. If a real-valued solution of Eq. (56) 701 exists, the system can generate the oscillation. Its frequency is 702 independent of $\Delta \psi$ and may be computed as follows: The I 703 neuron spikes at the beginning of the scenario and is reset. The 704 generated spike arrives at the I neuron at time $\tau$ and induces an 705 instantaneous change of the phase $\varphi_{I}$ from $\tau$ to $H_{I}\left(\tau, \varepsilon_{I \rightarrow I}\right)$.
To reach threshold and spike again, the I neuron needs the time 706 $\Theta_{I}-H_{I}\left(\tau, \varepsilon_{I \rightarrow I}\right)$. The period of the oscillation is the sum of 707 the two times and the oscillation frequency is given by

$$
f=\left[\tau+\Theta_{I}-H_{I}\left(\tau, \varepsilon_{I \rightarrow I}\right)\right]^{-1} .
$$

In a "pure ING" rhythm, the $\varepsilon_{E \rightarrow I}$ connection is deleted. 709 While the E neuron may still spike, it does not influence the I 710 neuron, such that its dynamics are the same as if the E neuron 711 were suppressed. We can thus derive the oscillation frequency 712 of the pure ING rhythm in the same manner as above and it is 713 also given by Eq. (57).

\section{Scenario 2 (a scenario leading to ING)}

In scenario 2 the I neuron spikes, followed by the E neuron 716 within time interval $\tau$; cf. Fig. 4(b). This happens, if before the 717 interaction

$$
-\tau<\Delta \psi<0
$$

The I and E phases at the start of the interaction sequence are 719

$$
\begin{gathered}
\psi_{I}=0, \\
\psi_{E}=\Delta \psi,
\end{gathered}
$$

respectively. The interaction sequence consists of sending and 720 receiving an I and an E spike. First, at $t=0$, the I neuron 721 sends a spike and resets, then the E neuron spikes and resets, 722 before the I spike arrives. The reset of the I neuron implies 723 that $\varphi_{I}$ equals $\tau$ when it receives its own, self-inhibitory spike. 724 Since the E spike has a conduction delay $\tau$ as well, but is sent 725 $-\psi_{E}=-\Delta \psi$ after the I spike, the E spike arrives at the I ${ }_{726}$ neuron at $\tau-\Delta \psi$, i.e., $-\Delta \psi$ after the self-inhibitory spike. 727 The I phase thus proceeds for $-\Delta \psi$ after the processing of 728 the I spike before the E spike arrives. This arrival also marks 729 the end of the interaction sequence. Taken together, the phase 730 $\tilde{\varphi}_{I}$ directly after the interaction sequence (i.e., directly after 731 receiving the $\mathrm{E}$ spike) reads with the interaction function $H_{I} 732$ of the I neuron

$$
\widetilde{\varphi}_{I}=H_{I}\left(H_{I}\left(\tau, \varepsilon_{I \rightarrow I}\right)-\Delta \psi, \varepsilon_{E \rightarrow I}\right),
$$

thus

$$
\widetilde{\psi}_{I}=\widetilde{\varphi}_{I}-\Theta_{I}=H_{I}\left(H_{I}\left(\tau, \varepsilon_{I \rightarrow I}\right)-\Delta \psi, \varepsilon_{E \rightarrow I}\right)-\Theta_{I} .
$$

We may assume $H_{I}\left(H_{I}\left(\tau, \varepsilon_{I \rightarrow I}\right)-\Delta \psi, \varepsilon_{E \rightarrow I}\right)<\Theta_{I}$; i.e., the 735 I neuron does not spike upon arrival of the E spike, since a ${ }_{736}$ regular oscillation where scenario 2 begins again at its very 737 end would require the $\mathrm{E}$ neuron to have an intrinsic period 738 smaller than or equal to $2 \tau$, which we excluded (the duration of 739 scenario 2 is at most $2 \tau$ and the E neuron would need to reach its 740 original phase again after its reset despite the inhibitory input). 741 The E neuron is reset at the time $t=-\Delta \psi$ after the time of 742 the I neuron's spike at $t=0$. It therefore has the phase $\tau-{ }_{743}$ $(-\Delta \psi)=\tau+\Delta \psi$ when the input from the I neuron arrives. 744 The I spike changes the phase of the E neuron to $H_{E}\left(\tau+{ }_{745}\right.$ $\left.\Delta \psi, \varepsilon_{I \rightarrow E}\right)$, where $H_{E}$ is the transfer function of the E neuron. 746 Thereafter, the $\mathrm{E}$ neuron evolves freely (since $\varepsilon_{E \rightarrow E}=0$ ) ${ }_{747}$ for a time $-\Delta \psi$ until the end of the interaction sequence at 748 
$t=(\tau-\Delta \psi)^{+}$. The phases then read

$$
\begin{gathered}
\widetilde{\varphi}_{E}=H_{E}\left(\tau+\Delta \psi, \varepsilon_{I \rightarrow E}\right)-\Delta \psi \\
\widetilde{\psi}_{E}=\widetilde{\varphi}_{E}-\Theta_{E}=H_{E}\left(\tau+\Delta \psi, \varepsilon_{I \rightarrow E}\right)-\Delta \psi-\Theta_{E} .
\end{gathered}
$$

Taken together,

$$
\Delta \widetilde{\psi}=\underbrace{H_{E}\left(\tau+\Delta \psi, \varepsilon_{I \rightarrow E}\right)-H_{I}\left(H_{I}\left(\tau, \varepsilon_{I \rightarrow I}\right)-\Delta \psi, \varepsilon_{E \rightarrow I}\right)-\Delta \psi-\Delta \Theta}_{=: G(\Delta \psi)} .
$$

Our considerations result again in an iteration map $G$, which maps the difference of the shifted phases before the interaction 751 sequence to the difference of the shifted phases thereafter.

Scenario 2 can repeat to give rise to regular oscillations. The underlying phase dynamics then satisfy

$$
G(\Delta \psi)=\Delta \psi
$$

Solving for $\Delta \psi$ allows us to determine the dynamics. If the E and I neurons are both LIF neurons, Eqs. (66) and (20) yield

$$
\begin{aligned}
\Delta \psi= & \ln \left\{\frac{e^{-\tau}-e^{-H_{\mathrm{LF}}\left(\tau, \varepsilon_{I \rightarrow I} ; \Theta_{I}\right)-\Delta \Theta}}{2 e^{-\Delta \Theta} \Gamma\left(\Theta_{E}, \varepsilon_{I \rightarrow E}\right)}\right. \\
& \left. \pm \frac{\sqrt{\left[e^{-H_{\mathrm{LF}}\left(\tau, \varepsilon_{I \rightarrow I} ; \Theta_{I}\right)-\Delta \Theta}-e^{-\tau}\right]^{2}+4 e^{-\Delta \Theta} \Gamma\left(\Theta_{E}, \varepsilon_{I \rightarrow E}\right) \Gamma\left(\Theta_{I}, \varepsilon_{E \rightarrow I}\right)}}{2 e^{-\Delta \Theta} \Gamma\left(\Theta_{E}, \varepsilon_{I \rightarrow E}\right)}\right\}-\Delta \Theta,
\end{aligned}
$$

755 where $\Gamma(\Theta, \varepsilon)$ is defined as

$$
\Gamma(\Theta, \varepsilon):=\left(1-e^{-\Theta}\right) \varepsilon .
$$

756 If the I neuron is the sine neuron, Eq. (39) has to be inserted for ${ }_{757} H_{I}$ in Eq. (65). We note that the I spike arrives at the I neuron 758 at the phase $\varphi_{I}=\tau$, which is in the first branch of the inverse 759 rise function, $\left.\varphi_{I}=\tau \in\right] 0, \Theta_{I} / 2$ [, because we assume that the 760 intrinsic period of the neuron is longer than $2 \tau$. The input thus 761 advances the phase and the first line of Eq. (39) will be used 762 to write out $H_{I}\left(\tau, \varepsilon_{I \rightarrow I}\right)$. In contrast, the $\mathrm{E}$ spike can arrive at 763 a phase of the I neuron in the first branch $\left.\varphi_{I} \in\right] 0, \Theta_{I} / 2[$ or 764 in the second branch $\left.\varphi_{I} \in\right] \Theta_{I} / 2, \Theta_{I}\left[\right.$ or at $\varphi_{I}=\Theta_{I} / 2$, so it 765 either delays or advances the phase or leaves it unchanged and 766 the first or second or third line of Eq. (39) applies to the outer ${ }_{767} H_{I}$ in $H_{I}\left(H_{I}\left(\tau, \varepsilon_{I \rightarrow I}\right)-\Delta \psi, \varepsilon_{E \rightarrow I}\right)$, depending on the value 768 of $H_{I}\left(\tau, \varepsilon_{I \rightarrow I}\right)-\Delta \psi$.

769 If a real-valued solution $\Delta \psi$ of Eq. (66) exists, the network 770 can generate a regular oscillation characterized by repeated 771 occurrence of scenario 2. The oscillation frequency can be 772 determined directly from the dynamics of the $\mathrm{E}$ neuron in 773 terms of $\Delta \psi$. We start at the time when the $\mathrm{E}$ neuron spikes 774 and is reset. After a time $\tau+\Delta \psi$ the inhibitory input from 775 the I neuron arrives; cf. Eqs. (63) and (64) and the paragraph 776 preceding them. The phase of the $\mathrm{E}$ neuron is changed to $777 H_{\mathrm{LIF}}\left(\tau+\Delta \psi, \varepsilon_{I \rightarrow E} ; \Theta_{E}\right)$ and it takes the $\mathrm{E}$ neuron the time $778 \Theta_{E}-H_{\mathrm{LIF}}\left(\tau+\Delta \psi, \varepsilon_{I \rightarrow E} ; \Theta_{E}\right)$ to spike again and complete 779 the period. Summing the two times up yields the oscillation 780 period and therewith the oscillation frequency of scenario 7812 ING,

$$
f(\Delta \psi)=\left[\tau+\Delta \psi+\Theta_{E}-H_{\mathrm{LIF}}\left(\tau+\Delta \psi, \varepsilon_{I \rightarrow E} ; \Theta_{E}\right)\right]^{-1} .
$$

\section{E. Scenario 3 (a scenario leading to ING)}

783 In scenario 3, first the E neuron spikes and then the I neuron, 784 before the spike from the E neuron arrives. This scenario occurs for

$$
0 \leqslant \Delta \psi<\tau
$$

The E neuron is leading, so the I and E phases at the start of 786 the interaction sequence read

$$
\begin{gathered}
\psi_{I}=-\Delta \psi, \\
\psi_{E}=0,
\end{gathered}
$$

respectively. At time $t=0$, the E neuron sends its spike and is $\quad 788$ reset; at time $\Delta \psi$, the I neuron sends its spike and is reset. The I 789 neuron thus receives the $\mathrm{E}$ spike while having a phase $\tau-\Delta \psi \quad 790$ at time $\tau$. Processing of the $\mathrm{E}$ spike by the I neuron yields 791 $H_{I}\left(\tau-\Delta \psi, \varepsilon_{E \rightarrow I}\right)$ and subsequent time evolution until the 792 receiving of the I spike by both the $\mathrm{E}$ and I neurons adds $\Delta \psi$ to $\quad 793$ the phase. We may assume $H_{I}\left(\tau-\Delta \psi, \varepsilon_{E \rightarrow I}\right)+\Delta \psi<\Theta_{I} \quad 794$ and thus exclude direct generation of a spike of the I neuron 795 because of the arrival of the spike from the E neurons, since 796 such a spike would break a regular oscillation. Accounting for 797 the I spike that arrives at the $\mathrm{E}$ and I neurons at time $\tau+\Delta \psi, \quad 798$ we obtain at the end of the scenario

and

$$
\begin{gathered}
\tilde{\varphi}_{E}=H_{E}\left(\tau+\Delta \psi, \varepsilon_{I \rightarrow E}\right), \\
\widetilde{\psi}_{E}=H_{E}\left(\tau+\Delta \psi, \varepsilon_{I \rightarrow E}\right)-\Theta_{E} .
\end{gathered}
$$


We conclude

$$
\Delta \widetilde{\psi}=\underbrace{H_{E}\left(\tau+\Delta \psi, \varepsilon_{I \rightarrow E}\right)-H_{I}\left(H_{I}\left(\tau-\Delta \psi, \varepsilon_{E \rightarrow I}\right)+\Delta \psi, \varepsilon_{I \rightarrow I}\right)-\Delta \Theta}_{=: G(\Delta \psi)} .
$$

Scenario 3 can repeat to give rise to regular oscillations. As before, if a real-valued solution of

$$
G(\Delta \psi)=\Delta \psi
$$

exists, the network can generate the oscillations and the solution $\Delta \psi$ specifies the underlying phase dynamics. The oscillation ${ }^{803}$ frequency can be determined directly from the dynamics of the $\mathrm{E}$ neuron in terms of $\Delta \psi$. At the beginning of the 804 scenario, the E neuron spikes and at the end the E neuron's phase is given by Eq. (75). It thus spikes again after a time 805 $\Theta_{E}-H_{\mathrm{LIF}}\left(\tau+\Delta \psi, \varepsilon_{I \rightarrow E} ; \Theta_{E}\right)$ to complete the oscillation cycle. The period of the oscillation is the sum of the duration 806 $\tau+\Delta \psi$ of the interaction sequence and the time to complete the cycle, such that the oscillation frequency is given by

$$
f(\Delta \psi)=\left[\tau+\Delta \psi+\Theta_{E}-H_{\mathrm{LIF}}\left(\tau+\Delta \psi, \varepsilon_{I \rightarrow E} ; \Theta_{E}\right)\right]^{-1} .
$$

When the $\mathrm{E}$ and I neurons are LIF neurons, Eq. (78) yields

$$
\Delta \psi=\ln \left\{\frac{\Gamma\left(\Theta_{I}, \varepsilon_{I \rightarrow I}\right)+e^{-\tau+\Delta \Theta}-e^{-\tau}}{2 \Gamma\left(\Theta_{E}, \varepsilon_{I \rightarrow E}\right)} \pm \frac{\sqrt{\left[e^{-\tau}-\Gamma\left(\Theta_{I}, \varepsilon_{I \rightarrow I}\right)-e^{-\tau+\Delta \Theta}\right]^{2}+4 \Gamma\left(\Theta_{E}, \varepsilon_{I \rightarrow E}\right) \Gamma\left(\Theta_{I}, \varepsilon_{E \rightarrow I}\right) e^{\Delta \Theta}}}{2 \Gamma\left(\Theta_{E}, \varepsilon_{I \rightarrow E}\right)}\right\}-\Delta \Theta
$$

809 where $\Gamma(\Theta, \varepsilon)$ is given by Eq. (68). Placing $\Delta \psi$ given in 838 that the received input is suprathreshold is then

810 Eq. (80) into Eq. (79) yields the frequency of the oscillation.

811 If the I neuron is the sine neuron, the $\mathrm{E}$ spike arrives at 812 the I neuron at a phase that is always within the first branch 813 of the inverse rise function, i.e., within ]0, $\Theta_{I} / 2$ [, because we 814 assume that the intrinsic period of the neurons is longer than ${ }_{815} 2 \tau . H_{I}\left(\tau-\Delta \psi, \varepsilon_{E \rightarrow I}\right)$ in Eq. (77) is then explicitly defined 816 by the first line of Eq. (39) and the excitatory input delays the 817 phase of the I neuron. The I spike thus also always arrives at 818 the I neuron at a phase within the first branch and advances the 819 phase.

\section{$820 \quad$ F. Scenario 4 (a scenario leading to PING)}

821

In scenario 4, the E neuron spikes first, followed by the I 22 neuron, which spikes due to suprathreshold excitatory input ${ }_{823}$ from the E neuron [cf. Fig. 4(b)]. We note that the scenario ${ }_{824}$ does not occur if the I neuron is a sine neuron because sine 825 neurons cannot be suprathresholdly excited as the required 826 input strength would be infinite [cf. derivation of Eqs. (38) 827 and (39)]. In scenario 4 the E neuron spikes at $t=0$, so the I ${ }_{828}$ and $\mathrm{E}$ phases at the start of the interaction sequence, at $t=0$, $829 \mathrm{read}$

$$
\begin{gathered}
\psi_{I}=-\Delta \psi, \\
\psi_{E}=0,
\end{gathered}
$$

830 respectively. For scenario $4, \Delta \psi$ must satisfy

$$
\tau \leqslant \Delta \psi \leqslant \Theta_{I}+\tau-H_{I}\left(\Theta_{I},-\varepsilon_{E \rightarrow I}\right) .
$$

831 The left-hand side inequality guarantees that the I neuron 832 does not spike before the $\mathrm{E}$ spike arrives. The right-hand 833 side inequality guarantees that the I neuron is at the time of 834 arrival of the excitatory input from the E neuron sufficiently 835 near the threshold to receive suprathreshold excitation: The E 836 spike arrives at time $t=\tau$ where the I neuron has phase $\psi_{I}=$ ${ }_{837}-\Delta \psi+\tau$ equivalent to $\varphi_{I}=\Theta_{I}-\Delta \psi+\tau$. The condition

$$
U_{I}\left(\Theta_{I}-\Delta \psi+\tau\right)+\varepsilon_{E \rightarrow I} \geqslant U_{I}\left(\Theta_{I}\right)=\Theta_{V, I} .
$$

We assume that $U_{I}(\varphi)$ is strictly monotonically increasing in 839 the relevant range near the threshold, such that $U_{I}^{-1}$ exists and 840 is strictly monotonically increasing. We can then apply it to 841 Eq. (84) maintaining the direction of the inequality:

$$
\begin{aligned}
\Theta_{I}-\Delta \psi+\tau & \geqslant U_{I}^{-1}\left(U_{I}\left(\Theta_{I}\right)-\varepsilon_{E \rightarrow I}\right), \\
& =H_{I}\left(\Theta_{I},-\varepsilon_{E \rightarrow I}\right) .
\end{aligned}
$$

Isolating $\Delta \psi$ yields

$$
\Delta \psi \leqslant \Theta_{I}+\tau-H_{I}\left(\Theta_{I},-\varepsilon_{E \rightarrow I}\right),
$$

which is the right-hand side inequality of Eq. (83).

The scenario now unfolds as follows: The E neuron sends 845 its spike and resets and the I neuron receives the E spike 846 at $t=\tau$. The excitatory input brings the I neuron above its 847 threshold, such that it spikes and resets subsequently. At $t=2 \tau \quad{ }_{848}$ both neurons receive the I spike. Due to the suprathreshold 849 excitation the precise value of the I phase when the E spike 850 arrives is irrelevant for the final phase. When the I neuron 851 receives the self-inhibitory I spike at the end of the interaction 852 sequence its phase is always $\varphi_{I}=\tau$, so

$$
\begin{gathered}
\widetilde{\varphi}_{I}=H_{I}\left(\tau, \varepsilon_{I \rightarrow I}\right), \\
\widetilde{\psi}_{I}=H_{I}\left(\tau, \varepsilon_{I \rightarrow I}\right)-\Theta_{I} .
\end{gathered}
$$

Since the E neuron was reset at $t=0^{+}$and evolves freely until 854 it receives the I spike at $t=2 \tau$,

$$
\begin{gathered}
\widetilde{\varphi}_{E}=H_{E}\left(2 \tau, \varepsilon_{I \rightarrow E}\right), \\
\widetilde{\psi}_{E}=H_{E}\left(2 \tau, \varepsilon_{I \rightarrow E}\right)-\Theta_{E} .
\end{gathered}
$$


The phase difference $\Delta \widetilde{\psi}$ after the interaction sequence thus reads

$$
\Delta \widetilde{\psi}=\underbrace{H_{E}\left(2 \tau, \varepsilon_{I \rightarrow E}\right)-H_{I}\left(\tau, \varepsilon_{I \rightarrow I}\right)-\Delta \Theta}_{=: G(\Delta \psi)} .
$$

858 859

Scenario 4 can also repeat to give rise to regular oscillations. The underlying phase dynamics then satisfy

$$
G(\Delta \psi)=\Delta \psi .
$$

860 Solving for $\Delta \psi$ yields

$$
\Delta \psi=\ln \left[\frac{e^{-\tau}-\Gamma\left(\Theta_{I}, \varepsilon_{I \rightarrow I}\right)}{e^{-2 \tau}-\Gamma\left(\Theta_{E}, \varepsilon_{I \rightarrow E}\right)}\right]-\Delta \Theta
$$

861 (both neurons are LIF neurons for the scenario to occur). ${ }_{862}$ If the solution is real-valued, the network can generate the ${ }_{863}$ oscillation. The oscillation period can be determined directly 864 from the dynamics of the E neuron. At the beginning of the 865 scenario, the E neuron sends a spike and is reset. The I spike 866 arrives after a time $2 \tau$ at the $\mathrm{E}$ neuron. The $\mathrm{E}$ phase at this point 867 is $2 \tau$, which changes to $H_{E}\left(2 \tau, \varepsilon_{I \rightarrow E}\right)$. The $\mathrm{E}$ neuron will thus 868 spike next after a time $\Theta_{E}-H_{E}\left(2 \tau, \varepsilon_{I \rightarrow E}\right)$. Summing the two 869 times up yields the oscillation period and the frequency

$$
f=\left[2 \tau+\Theta_{E}-H_{E}\left(2 \tau, \varepsilon_{I \rightarrow E}\right)\right]^{-1} .
$$

870 Inserting Eq. (20) yields

$$
f=\left\{2 \tau+\Theta_{E}+\ln \left[e^{-2 \tau}-\Gamma\left(\Theta_{E}, \varepsilon_{I \rightarrow E}\right)\right\},\right.
$$

871 where $\Gamma$ is defined in Eq. (68). We note that due to the 872 suprathreshold excitation of the I neuron, the frequency is 873 independent of $\Delta \psi$ in contrast to oscillations generated by 874 other scenarios.

875

876 877 the E neuron spiking. It occurs for

$$
\Theta_{I}+\tau-H_{I}\left(\Theta_{I},-\varepsilon_{E \rightarrow I}\right)<\Delta \psi ;
$$

878 the phases of the $\mathrm{I}$ and $\mathrm{E}$ neurons at the start of the interaction 879 sequence are

$$
\begin{gathered}
\psi_{I}=-\Delta \psi, \\
\psi_{E}=0,
\end{gathered}
$$

${ }_{856}$ respectively. The E neuron sends a spike at the beginning of ${ }_{880}$ ${ }_{857}$ the sequence, which is received by the I neuron at $t=\tau$. Since ${ }_{881}$ the I neuron does not spike, this marks the end of the scenario. 882 The phase $\varphi_{I}$ of the I neuron at receiving is

$$
\varphi_{I}=\Theta_{I}-\Delta \psi+\tau .
$$

After the receiving, at the end of the scenario the phases read 884

$$
\begin{gathered}
\tilde{\varphi}_{I}=H_{I}\left(\Theta_{I}-\Delta \psi+\tau, \varepsilon_{E \rightarrow I}\right), \\
\tilde{\psi}_{I}=H_{I}\left(\Theta_{I}-\Delta \psi+\tau, \varepsilon_{E \rightarrow I}\right)-\Theta_{I} .
\end{gathered}
$$

The condition $\Theta_{I}+\tau-H_{I}\left(\Theta_{I},-\varepsilon_{E \rightarrow I}\right)<\Delta \psi$ implies 885 $H_{I}\left(\Theta_{I}-\Delta \psi+\tau, \varepsilon_{E \rightarrow I}\right)<\Theta_{I}$, such that the I neuron does 886 not spike. The E neuron evolves freely after its reset at $t=0^{+},{ }_{887}$ so

$$
\begin{gathered}
\widetilde{\varphi}_{E}=\tau, \\
\widetilde{\psi}_{E}=\tau-\Theta_{E},
\end{gathered}
$$

which yields

$$
\Delta \widetilde{\psi}=\underbrace{\tau-H_{I}\left(\Theta_{I}+\tau-\Delta \psi, \varepsilon_{E \rightarrow I}\right)-\Delta \Theta}_{=: G(\Delta \psi)} .
$$

\section{H. Alternation between scenarios 5 and 1}

In scenarios 2, 3, and 4 both neurons spike such that regular 891 oscillations must be generated by repeating a single scenario. 892 In contrast, scenarios 1 and 5 have to alternate to generate a ${ }_{893}$ regular oscillation. In this section, we derive the phase-locking 894 equation and the frequency for this type of oscillation. Without 895 loss of generality, we assume that the spiking pattern begins 896 with scenario 5 and scenario 1 follows. $\Delta \psi$ at $t=0$ has to ${ }_{897}$ satisfy Eq. (96) for scenario 5 to occur. $\Delta \widetilde{\psi}$ after scenario 5898 given in Eq. (104) has to satisfy Eq. (46) for scenario 1 to occur. 899 Thus, alternation between scenarios 5 and 1 occurs for

$$
2 \tau-\Delta \Theta \leqslant H_{I}\left(\Theta_{I}+\tau-\Delta \psi, \varepsilon_{E \rightarrow I}\right) .
$$

Composing the maps Eqs. (104) and (55), we obtain

$$
\Delta \widetilde{\psi}=\underbrace{H_{E}\left(\Theta_{I}+2 \tau-H_{I}\left(\Theta_{I}+\tau-\Delta \psi, \varepsilon_{E \rightarrow I}\right), \varepsilon_{I \rightarrow E}\right)-H_{I}\left(\tau, \varepsilon_{I \rightarrow I}\right)-\Delta \Theta}_{=: G^{2}(\Delta \psi)} .
$$

902 Note that now we have two iterations of the map $G$, which 903 maps the difference of the shifted phases before scenario 5 904 to the difference between the shifted phases after scenario 1 . 905 To determine the phase underlying the oscillation, we need to 906 solve

$$
\Delta \psi=G^{2}(\Delta \psi)
$$

907 for $\Delta \psi$. If a real-valued solution $\Delta \psi$ exists, the network can 908 generate the oscillations. Their frequency can be derived in terms of $\Delta \psi$ : In the initial scenario 5, the $\mathrm{E}$ neuron spikes 909 at time $t=0$. The phases $\varphi_{E}$ and $\varphi_{I}$ at the scenario's end are 910 given by Eqs. (100) and (102), respectively. The duration of the 911 scenario is $\tau$. Initializing scenario 1 , the I neuron spikes after 912 a time $\Theta_{I}-H_{I}\left(\Theta_{I}+\tau-\Delta \psi, \varepsilon_{E \rightarrow I}\right)$. The output from the I ${ }_{913}$ neuron arrives at the $E$ neuron at the phase $\varphi_{E}=2 \tau+\Theta_{I}-{ }_{914}$ $H_{I}\left(\Theta_{I}+\tau-\Delta \psi, \varepsilon_{E \rightarrow I}\right)$ of the E neuron and causes it to jump 915 to $H_{E}\left(2 \tau+\Theta_{I}-H_{I}\left(\Theta_{I}+\tau-\Delta \psi, \varepsilon_{E \rightarrow I}\right), \varepsilon_{I \rightarrow E}\right)$. The du- 916 ration of scenario 1 is $\tau$ as well. The $E$ neuron needs a time ${ }_{917}$ 
200 of

${ }_{921} 1$ thus equals $2 \tau+\Theta_{E}+\Theta_{I}-H_{I}\left(\Theta_{I}+\tau-\Delta \psi, \varepsilon_{E \rightarrow I}\right)-$ $922 H_{E}\left(2 \tau+\Theta_{I}-H_{I}\left(\Theta_{I}+\tau-\Delta \psi, \varepsilon_{E \rightarrow I}\right), \varepsilon_{I \rightarrow E}\right)$ and the os${ }_{923}$ cillation frequency is

$$
\begin{aligned}
f(\Delta \psi)= & {\left[2 \tau+\Theta_{E}+\Theta_{I}-H_{I}\left(\Theta_{I}+\tau-\Delta \psi, \varepsilon_{E \rightarrow I}\right)\right.} \\
& -H_{E}\left(2 \tau+\Theta_{I}-H_{I}\left(\Theta_{I}+\tau\right.\right. \\
& \left.\left.\left.-\Delta \psi, \varepsilon_{E \rightarrow I}\right), \varepsilon_{I \rightarrow E}\right)\right]^{-1}
\end{aligned}
$$

\section{4}

925

926

927$$
928
$$$$
929
$$

$$
930
$$$$
930
$$
931 has been described extensively in the literature (cf., e.g., $\left.{ }_{932}[53,54,56]\right)$. Alternatively, we can consider networks without ${ }_{933} \mathrm{E}$ to I coupling; they generate the same I dynamics even if 934 the E neuron continues to spike. Similarly well studied (cf., 935 e.g., $[54,56,72])$ is the straightforward PING condition, where 936 a relatively large drive to the $\mathrm{E}$ neuron causes it to spike ${ }_{937}$ periodically. These E spikes generate spikes in the I neuron, 938 which has small drive and would remain rather inactive without 939 the input from the E neuron. In this paper we will focus on 940 situations where ING and PING are in competition since both 941 the E and I neurons have comparably strong drives and all 942 relevant couplings are present. However, we will consider ${ }_{943}$ the above-mentioned straightforward "pure ING" and "pure ${ }_{944}$ PING" rhythms for comparison. As described in Sec. IV, there 945 are 5 possible scenarios for relative spiking of the E and I 946 neurons. These can-alone or in combination-give rise to 947 regular oscillations, more precisely to ING and PING rhythms. 948 Scenarios 2 and 3, in which the I neuron spikes due to its 949 intrinsic dynamics before the E input arrives, generate an ING 950 rhythm. Scenario 4 , in which the spike of the I neuron is 951 generated by the input from the E neuron instantaneously upon 952 its arrival, generates a PING rhythm. An oscillation generated 953 by scenarios 5 and 1 in alternation should be interpreted as ${ }_{954}$ PING rhythm, if the spike of the I neuron is generated shortly 955 after the input of the $E$ neuron, i.e., if the input from the $E$ 956 neuron basically generates the I spike. If the I spike occurs with 957 larger distance from the E spike, the character of the oscillation 958 becomes unclear. Because for the considered parameters our 959 simulations show spiking of the I neuron only shortly after the ${ }_{960} \mathrm{E}$ input (see Sec. VII for further discussion), for simplicity we ${ }_{961}$ denote all scenarios 5,1 generated oscillations as PING in the 962 following.
A. Global iteration map
${ }_{964}$ The local iteration maps derived in Sec. IV are valid for $965 \Delta \psi$ within a certain range, where the corresponding scenario 966 occurs. To analytically identify regular oscillations we gather 967 the local iteration maps into a global, piecewise defined 968 iteration map $G$, which maps the difference of the shifted ${ }_{969}$ phases $\Delta \psi$ to the difference of the shifted phases after the

next occurring interaction scenario. The global iteration map 970 consists of several sections, since the next interaction scenario 971 and thus the applicable map depend on the current difference 972 of the shifted phases [e.g., Fig. 4(b)]. Equations (46), (58), 973 (70), (83), and (96) specify the ranges, in which the different 974 scenarios occur, and thus the domains of the individual map 975 segments constituting $G$. Equations (55), (65), (77), (91), and 976 (104) give the corresponding maps. The regular oscillations 977 are reflected by fixed points of $G$ (scenarios 2,3 , and 4 ) and 978 $G^{2}$ (scenarios 5,1 in alternation).

\section{B. Phased locked oscillations in networks with type I $E$ and I neurons}

Figure 5(a) shows an example of an ING rhythm (scenario 982 2) in a network of two type I LIF neurons in standard phase 983 representation (cf. Sec. II). In this scenario, the I neuron (blue 984 trace) spikes just before spiking of the E neuron (red trace) 985 such that the inhibition from the I neuron to the E neuron 986 arrives after spiking of the E neuron. Figure 5(c) shows the 987 global iteration map $G$ for the same network parameters. The 988 panel displays the segments of the graph of $G$ in different 989 colors to highlight the five scenarios [see Fig. 4(b) for the color 990 labels]. The phase differences $\Delta \psi$ that satisfy $G(\Delta \psi)=\Delta \psi \quad 991$ are fixed points, which may be stable (if the absolute value of 992 the slope of the iteration map at the fixed point is less than 1) or 993 unstable (if the absolute value of the slope is larger than 1). The 994 only fixed point for $G$ in Fig. 5(c) is at the intersection of the 995 magenta segment (scenario 2) with the diagonal (black, slope 996 1) near $\Delta \psi=-0.2$. It is stable. Figure 5(e) shows the iteration 997 map after two periods, i.e., $G^{2}(\Delta \psi):=G(G(\Delta \psi))$. The thick 998 segment coloring of the curve indicates the scenarios occurring 999 in the first iteration [same as in panel (c)], while the thin curves 1000 highlight the scenarios in the second iteration. In both maps 1001 Figs. 5(c) and 5(e) the fixed point near $\Delta \psi=-0.2$ (repeated 1002 scenario 2) is the only one. It is stable and corresponds to the ${ }_{1003}$ ING rhythm displayed in panel (a). This fixed point is robust 1004 against variations in the drive to the $\mathrm{E}$ and I neurons and to 1005 changes in parameter values for synaptic connectivity. 1006

Figure 5(b) shows an example of a PING rhythm (scenario 1007 4) in a network with two type I LIF neurons in standard phase 1008 representation. The spike from the E neuron causes excitation 1009 of the I neuron above its spiking threshold, followed by a spike 1010 and reset of the I neuron. The global iteration map $G$ is shown 1011 in Fig. 5(d). There is a fixed point near $\Delta \psi=0.6$ where the 1012 red segment (scenario 4) crosses the diagonal. The segment 1013 is horizontal (slope zero). This means that the fixed point is 1014 stable and that the entire range of initial phase differences $\Delta \psi \quad 1015$ between roughly 0.4 and 0.9 is mapped to it exactly. This can 1016 also be directly seen from Eq. (91): The right-hand side is 1017 independent of $\Delta \psi$, such that the piece of the iteration map 1018 maps any initial relative phase in its domain to the same value. 1019 The second iteration map is shown in Fig. 5(f); we find only 1020 the same fixed point as in the first iteration map.

\section{Phased locked oscillations in networks with type I E and type II I neurons}

As explained in Sec. IV F, networks with the type II sine I ${ }_{1024}$ neuron cannot generate scenario 4. We therefore illustrate the 1025 
(a)

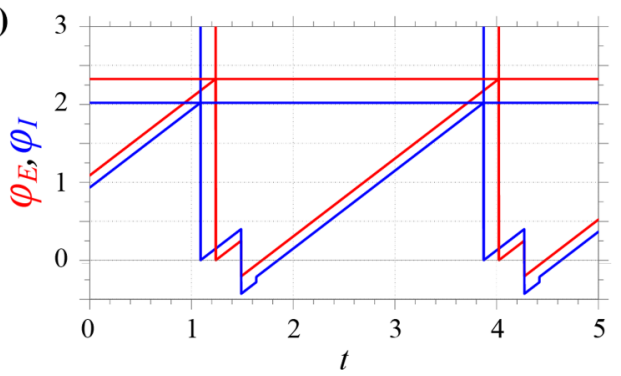

(c)

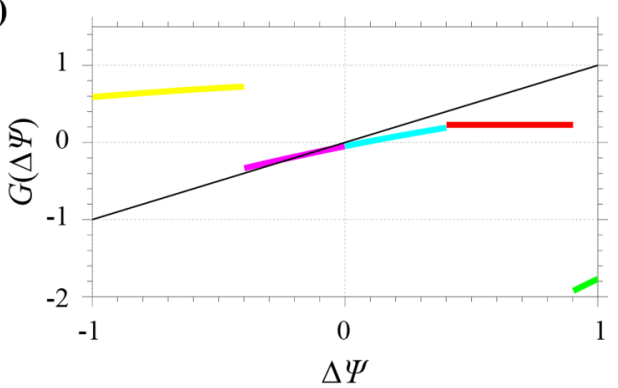

(e)

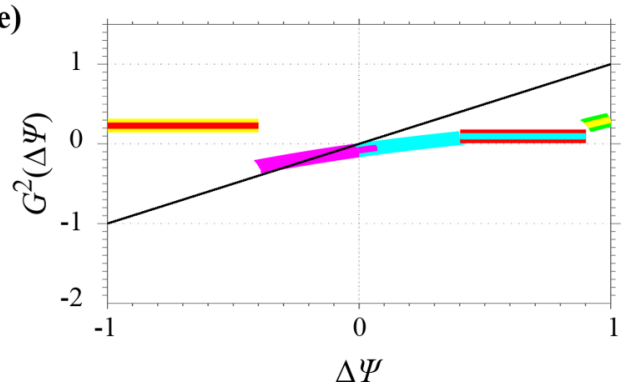

(b)

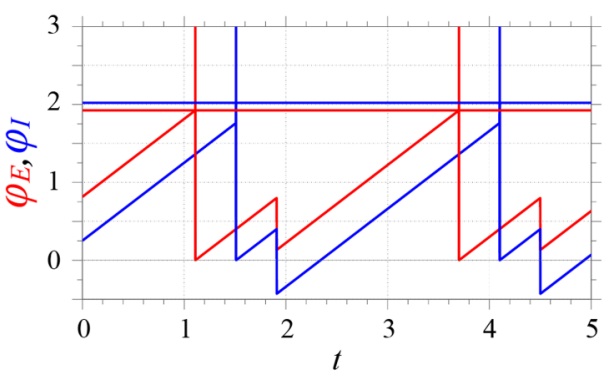

(d)

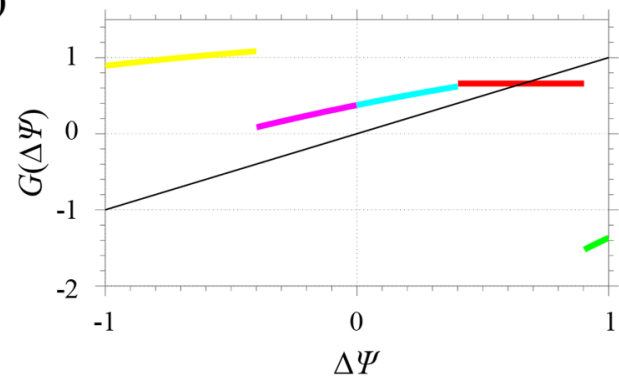

(f)

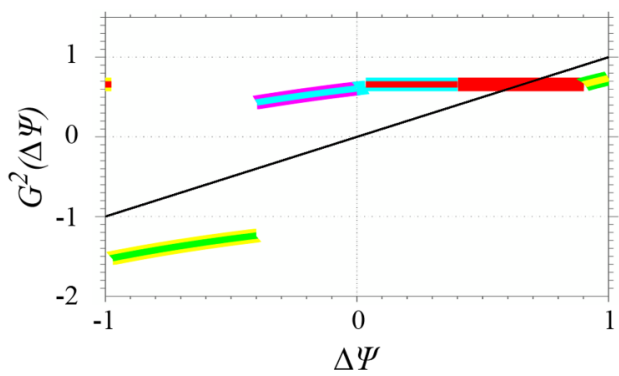

FIG. 5. ING and PING dynamics in a network of two type I (leaky integrate-and-fire) neurons. (a) ING dynamics (scenario 2 ) in phase representation. The panel shows $\varphi_{E}$ (red) and $\varphi_{I}$ (blue) versus time. Spikes are highlighted by upward vertical lines starting at the threshold. (b) PING dynamics (scenario 4) with suprathreshold excitation. (c) Iteration map $G$ with network parameters as in (a). Pieces of the map originating from different scenarios are highlighted by different colors [scenario 1: yellow, 2: magenta, 3: cyan, 4: red, 5: green; cf. frame colors in Fig. 4(b)]. There is a stable fixed point near $\Delta \psi=-0.2$ corresponding to the ING rhythm in (a). (d) Iteration map $G$ with network parameters as in (b). The stable fixed point near $\Delta \psi=0.7$ corresponds to the PING rhythm in (b). Panels (e) and (f) show the second iteration maps $G^{2}$, where the thick coloring of the segments indicates the first iteration also appearing in (c) and (d) and the thin coloring indicates the second. Parameter settings: $\varepsilon_{I \rightarrow E}=-0.5, \varepsilon_{E \rightarrow I}=0.1, \varepsilon_{I \rightarrow I}=-1.0$, and $\tau=0.4$; the drives to the $\mathrm{I}$ and $\mathrm{E}$ neurons are $1 / \Theta_{I}=0.495$ and $1 / \Theta_{E}=0.43$ for (a) and $1 / \Theta_{I}=0.495$ and $1 / \Theta_{E}=0.52$ for (b).

1026 dynamics of networks with an excitatory type I LIF neuron

and an inhibitory type II sine neuron with different scenarios than the dynamics of networks with two type I LIF neurons. We choose a scenario 3 ING rhythm and a scenarios 5,1 PING rhythm. We note that we observe for the considered parameters fixed points of $G$ in the domain of scenario 2; the purple curve (scenario 2) crosses the diagonal near $\Delta \psi=-0.2$ in Fig. 6(c) and near $\Delta \psi=-0.3$ in Fig. 6(d). However, the fixed points are unstable, as the absolute value of the slope of the iteration map $G$ is greater than 1 there. Consequently, the fixed points do not correspond to stable oscillations.

Figure 6(a) shows the ING dynamics generated by scenario 3. While the E neuron spikes just before sending of the I spike, as argued above this scenario does not belong to the class of PING, because spiking of the I neuron is not triggered by the E spike. The global iteration map $G$ is displayed in Fig. 6(c); it has a stable fixed point near $\Delta \psi=0.2$ in the domain of scenario 3 (intersection of the cyan curve with the diagonal). The results for the second iteration map are shown in Fig. 6(e) with the same stable fixed point near $\Delta \psi=0.2$ (repeated ${ }_{1045}$ scenario 3$)$.

Figure 6(b) shows phase dynamics that are generated by ${ }_{1047}$ alternation of scenarios 5 and 1 . We can clearly classify this ${ }_{1048}$ pattern as PING, since excitation from the E neuron brings the 1049 I neuron close to its threshold, which results in spiking of the I ${ }_{1050}$ neuron shortly thereafter. Figure 6(d) depicts the first iteration 1051 map $G$, which does not have a stable fixed point. In contrast, 1052 the second iteration map $G^{2}$ [Fig. 6(f)] has two stable fixed ${ }_{1053}$ points, reflecting the period 2 orbit that generates the PING ${ }_{1054}$ oscillation. They are located near $\Delta \psi=0.6$ and $\Delta \psi=-0.7 \quad 1055$ and correspond to alternating scenarios 5 and 1 and the phase ${ }_{1056}$ dynamics Fig. 6(b).

\section{PING-ING INTERACTIONS IN NETWORKS OF TWO OSCILLATORS}

We saw in the previous section that for suitable parameter 1060 values, our networks can generate either ING or PING rhythms. 1061 
(a)

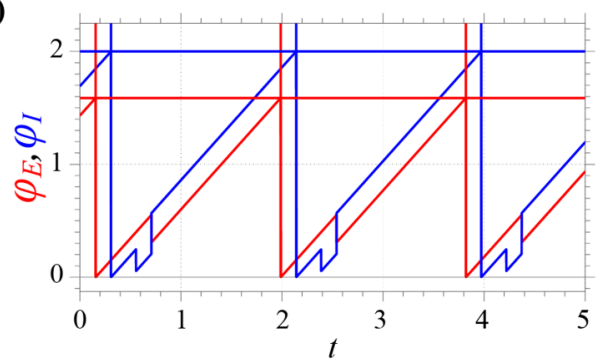

(c)

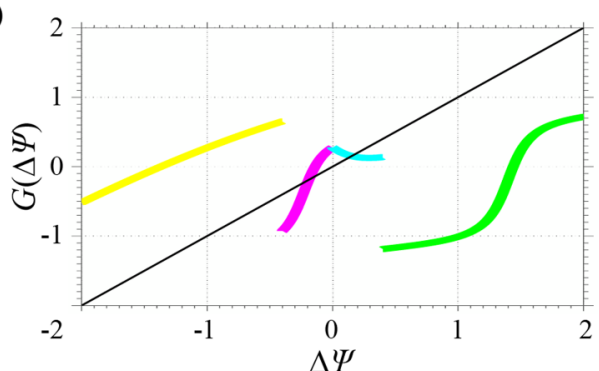

(e)

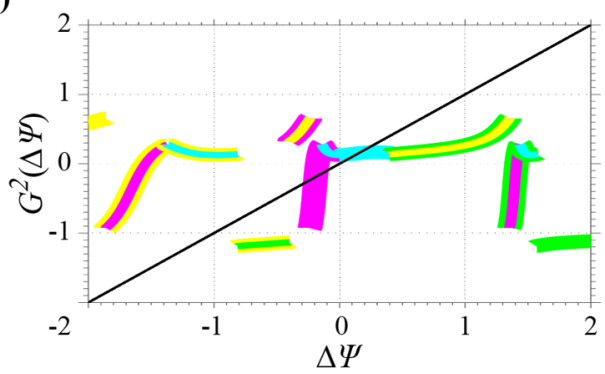

(b)

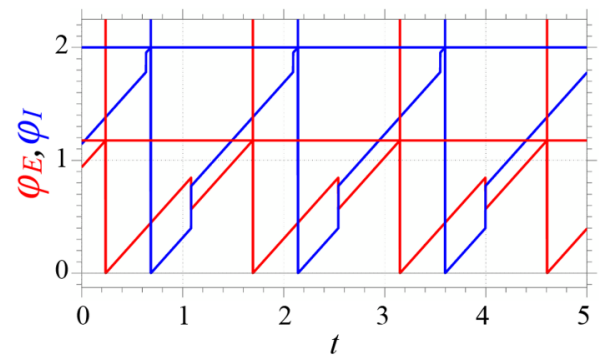

(d)

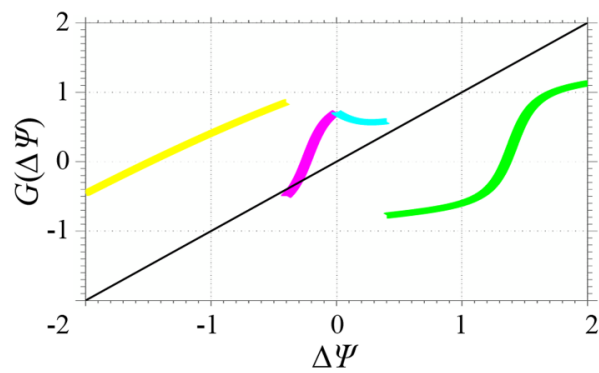

(f)

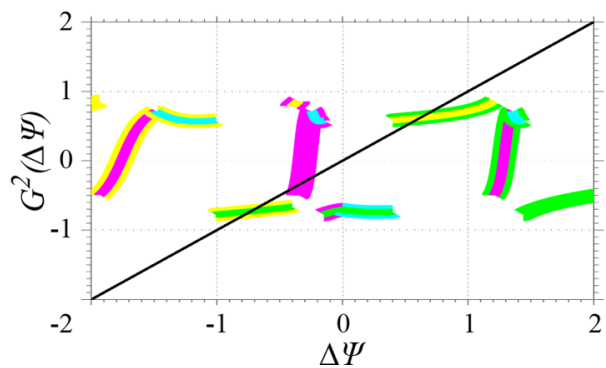

FIG. 6. ING and PING dynamics in a network of a type I (leaky integrate-and-fire) E neuron and a type II (sine) I neuron. (a) ING (scenario 3) and (b) PING (combination of scenarios 5 and 1) dynamics in phase representation. (c) and (d): Iteration maps $G$ for the same network parameters as used in (a) and (b), respectively. The stable fixed point near $\Delta \psi=0.2$ in (c) corresponds to the ING rhythm in (a). The other fixed point near $\Delta \psi=-0.2$ is unstable and corresponds to an unstable scenario 2 ING rhythm. (d) There is no fixed point of the first iteration map $G$ corresponding to the PING dynamics shown in panel (b), since they consist of a sequence of two scenarios and thus appear as a period 2 orbit in the iterations of $G$. The unstable fixed point near $\Delta \psi=-0.3$ in (d) corresponds to an unstable scenario 2 ING rhythm. Pieces of the map generated by different scenarios are highlighted by different colors as in Fig. 5, panels (c) and (d). (e) and (f): The second iteration maps $G^{2}$. The period 2 orbit of the PING rhythm in (b) is reflected by two fixed points in the second iteration map (f), in the domains of scenarios 1 and 5. Parameter settings: $\varepsilon_{I \rightarrow E}=-0.2, \varepsilon_{E \rightarrow I}=0.5, \varepsilon_{I \rightarrow I}=-0.42$, and $\tau=0.4$; the drives to the $\mathrm{I}$ and $\mathrm{E}$ neurons are $1 / \Theta_{I}=0.5$ and $1 / \Theta_{E}=0.63$ for (a) and $1 / \Theta_{I}=0.5$ and $1 / \Theta_{E}=0.85$ for (b).

${ }_{1062}$ In the following, we analyze how PING and ING rhythms 1063 compete to generate the network oscillation and how networks 1064 may switch from one rhythm to another when the values of the 1065 external drives change. We use "pure ING" and "pure PING" 1066 rhythms generated by reduced two-neuron networks, which do 1067 not allow for the generation of the other rhythm as reference. ${ }_{1068}$ This allows us to better understand the competition of PING 1069 and ING rhythms in the full network, which could in principle 1070 generate both rhythms. We express the external drive given to 1071 each neuron both for the LIF and sine neuron by the inverse of 1072 the period, i.e., by $1 / \Theta_{E}$ and $1 / \Theta_{I}$, since-in contrast to the 1073 LIF neuron - the sine neuron does not have an explicit external 1074 driving current variable.

\section{5}

1076 In "pure ING" networks the only excitatory input to the I 1077 neuron is the external drive, since the synaptic strength of the 1078 projection from the $\mathrm{E}$ to the I neuron is set to zero (cf. also 1079 [58]). The frequency of the pure ING rhythm is determined by the I drive and the self-inhibitory input with strength $\varepsilon_{I \rightarrow I} 1080$ arriving a time $\tau$ after reset of the I neuron; the frequency is 1081 explicitly given by Eq. (57).

In "pure PING" networks, the I drive is sufficiently small 1083 such that the I neuron has a much lower intrinsic period than the ${ }_{1084}$ E neuron. The circuit has a sufficiently strong projection from ${ }_{1085}$ the $\mathrm{E}$ to the I neuron that each $\mathrm{E}$ spike brings the membrane ${ }_{1086}$ potential of the I neuron above the threshold and elicits a spike ${ }_{1087}$ just as in scenario 4. The frequency of the pure PING rhythm ${ }_{1088}$ is determined by the $\mathrm{E}$ drive and the inhibitory input $\varepsilon_{I \rightarrow E}$ that ${ }_{1089}$ arrives after an interval $2 \tau$ after reset of the E neuron. The 1090 frequency is explicitly given by Eq. (95).

\section{B. Analysis of PING-ING interactions in networks with type I $E$ and I neurons}

We first study interactions between PING and ING rhythms 1094 for networks with two type I LIF neurons. The drives to the 1095 I neuron (I drive expressed by $1 / \Theta_{I}$ ) and to the E neuron (E 1096 drive expressed by $1 / \Theta_{E}$ ) vary; see Fig. 7. The blue surface ${ }_{1097}$ 
(a)

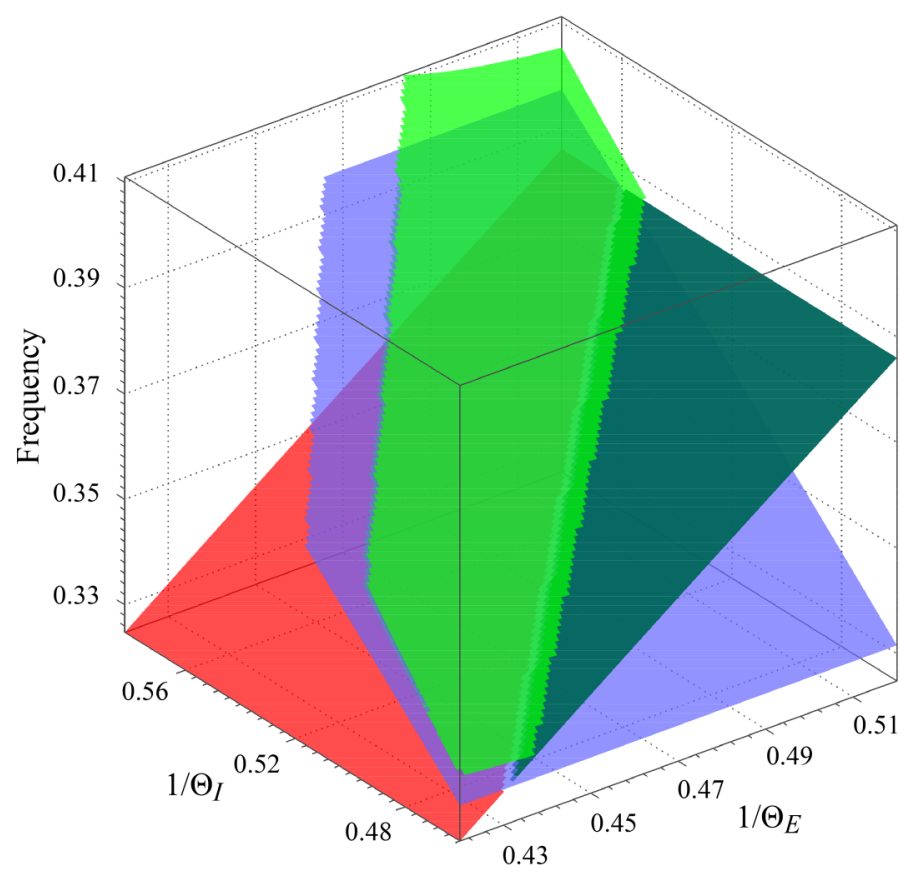

(b)

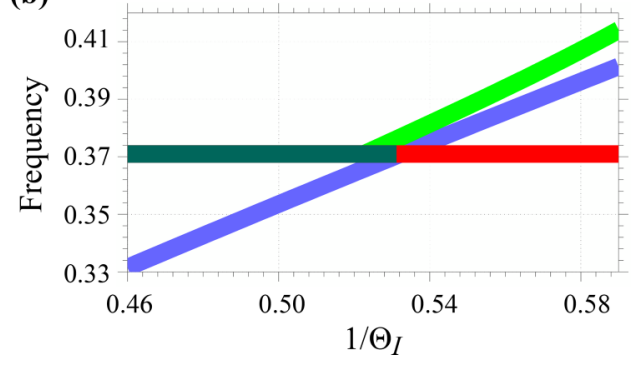

(c)

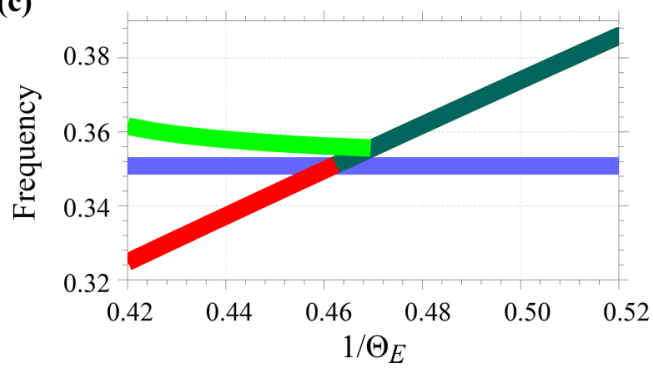

FIG. 7. Transitions between PING and ING in a network of two type I (leaky integrate-and-fire) neurons. The blue and red surfaces or curves show the oscillation frequencies of pure ING and pure PING rhythms, respectively. The green surfaces or curves show the frequency of oscillations in the full two-neuron network. Panel (a) displays the frequency of network oscillations versus the E and I drives (measured by intrinsic period ${ }^{-1}$ ). Termination of a surface in (a) occurs at parameters $1 / \Theta_{E}$ and $1 / \Theta_{I}$ where the highlighted network type does not yield any regular rhythm anymore. Panels (b) and (c) show cross sections of the surfaces given in (a). The drive at the I neuron (b) or at the E neuron (c) increases from left to right while the other drive is kept fixed. Light green curves show the frequency of the full network ING rhythm while dark green curves show the frequency of the full network PING rhythm. Parameter settings: $\varepsilon_{I \rightarrow E}=-0.5, \varepsilon_{E \rightarrow I}=0.1, \varepsilon_{I \rightarrow I}=-1.0$, and $\tau=0.4$; in (b) the drive to the E neuron is $1 / \Theta_{E}=0.495$ and in (c) the drive to the I neuron is $1 / \Theta_{I}=0.495$.

1098 in Fig. 7(a) shows the frequency of rhythmic spiking of the 1099 I neuron in pure ING networks. The red surface in Fig. 7(a) 1100 shows the frequency of rhythmic spiking of the E neuron in 1101 pure PING networks as a function of the $\mathrm{E}$ drive only. The 1102 green surface in Fig. 7(a) shows the frequency of rhythmic 1103 spiking for the full network schematically drawn in Fig. 4(a). 1104 The frequencies of the pure ING (blue surface) and of the full 1105 network (green surface) are not shown for some combinations 1106 of $1 / \Theta_{I}$ and $1 / \Theta_{E}$; these combinations do not elicit regular 1107 rhythms for scenarios 2, 3, and 4 and alternation of scenarios ${ }_{1108} 5$ and 1 for the displayed network type. Regular ING rhythms 1109 with suppressed E neuron (scenario 1 alone) are not generated 1110 either. The intersection of the surfaces in Fig. 7(a) with a plane 1111 of constant $E$ drive $\left(1 / \Theta_{E}=0.495\right)$ is shown in Fig. 7(b) and 1112 with a plane of constant I drive $\left(1 / \Theta_{I}=0.495\right)$ in Fig. $7(\mathrm{c})$.

1113 Figure 7(b) shows that for the range of comparably small I 1114 drive $1 / \Theta_{I}$ the rhythm of the full network is PING [scenario
4; dark green line in Fig. 7(b)]. The spiking pattern of the 1115 rhythm is the same as the spiking pattern of the pure PING ${ }_{1116}$ rhythm; cf. Fig. 5(b) for an example. The red line (pure PING) ${ }_{1117}$ and the green line (PING for the full network) in Fig. 7(b) 1118 thus overlap. The rhythm of the full network is PING, because 1119 the E neuron recovers from the inhibition sooner than the I 1120 neuron does and the E spike elicits spiking of the I neuron 1121 at its arrival. This also implies that when the full network 1122 generates PING, its frequency is higher than the frequency of ${ }_{1123}$ full network ING; otherwise the I neuron will spike by its own 1124 dynamics and consequently the full network generates ING. ${ }_{1125}$ Equation (95) shows that the frequency of this PING rhythm ${ }_{1126}$ (and the PING fixed point of the iteration map) does not depend ${ }_{1127}$ on the I drive $1 / \Theta_{I}$. When the I drive increases, there is a ${ }_{1128}$ bifurcation and a (stable) scenario 3 ING solution appears near ${ }_{1129}$ $1 / \Theta_{I}=0.52$ (light green curve): This ING solution lasts till 1130 near $1 / \Theta_{I}=0.56$, after which it switches to (stable) scenario $2{ }_{1131}$ 
ING. The frequency of the full-network ING rhythm increases with $1 / \Theta_{I}$. It stays higher than the frequency of pure ING 134 because the nonzero $\varepsilon_{E \rightarrow I}$ provides an additional excitatory 35 input to the I neuron and increases the frequency of the 36 rhythm. Interestingly, we find coexistence of PING and ING ${ }_{137}$ and bistability; cf. the range $0.52 \lesssim 1 / \Theta_{I} \lesssim 0.53$ in Fig. 7(b). ${ }_{1138}$ As $1 / \Theta_{I}$ increases further, the PING rhythm (dark green line) 1139 vanishes. If the network was oscillating in PING mode before, 1140 it will change to an ING rhythm and the oscillation frequency 1141 will increase in a jumplike manner.

1142 The reason for the vanishing of the PING mode is as 1143 follows: With increasing I drive, $\left|\psi_{I}\right|$ (the phase distance to 1144 the threshold $\Theta_{I}$ ) at arrival of the E spike becomes smaller 1145 until the $I$ neuron reaches $\Theta_{I}$ by its intrinsic dynamics at 1146 E-spike arrival. Beyond this point, there is no PING rhythm, 1147 as the I neuron spikes before E-spike arrival. The bifurcation 1148 point is at the crossing of the pure PING line (red) and the 1149 pure ING curve (blue): Since the I neuron reaches threshold 1150 from its own drive simultaneously with the E-spike arrival, 1151 the value of $\varepsilon_{E \rightarrow I}$ becomes irrelevant. At this bifurcation 1152 point, any input will generate suprathreshold excitation and 1153 be completely canceled due to the I neuron's reset such that 1154 also the oscillation frequencies of pure PING (large $\varepsilon_{E \rightarrow I}$ ) and 1155 pure ING $\left(\varepsilon_{E \rightarrow I}=0\right)$ agree.

1156 Taken together, we observe that the PING frequency is 1157 insensitive to changes in $1 / \Theta_{I}$, while the ING frequency 1158 increases with the drive. The PING rhythm vanishes when its 1159 frequency drops below that of the pure ING rhythm and the 1160 ING rhythm vanishes when its frequency drops below that of 1161 the PING rhythm. Since the ING rhythm of the full network 1162 has higher frequency than the pure ING rhythm, we have a 1163 region of coexistence. When the full network generates ING, its 1164 frequency is always higher than the frequency of full network 1165 PING. This is due to the fact that in ING the inhibition arrives 1166 at an E phase less than $2 \tau$ and thus [Fig. 2(c)] has a smaller 1167 phase-delaying impact than in PING, where it arrives at $2 \tau$ or 1168 later. We note that the slope of the light green curve is larger 1169 than the slope of the dark green line. In other words, the ING 1170 frequency is more sensitive to a change of the I drive $1 / \Theta_{I}$ 1171 than the insensitive PING frequency.

1172 Figure 7(c) shows the frequency of rhythms as we fix $1 / \Theta_{I}$ 1173 and vary $1 / \Theta_{E}$. For small $\mathrm{E}$ drive [e.g., $0.42 \lesssim 1 / \Theta_{E} \lesssim 0.46$ 1174 in Fig. 7(c)], the ING rhythm governs the dynamics of the 1175 full network: With our network parameters, it is the scenario 2 1176 ING rhythm for $0.42 \lesssim 1 / \Theta_{E} \lesssim 0.44$ and the scenario 3 ING 1177 rhythm for $0.44 \lesssim 1 / \Theta_{E} \lesssim 0.46$ (present for $0.44 \lesssim 1 / \Theta_{E} \lesssim$ 1178 0.47). As in Fig. 7(b), in Fig. 7(c) the full network ING rhythm $1179\left(\varepsilon_{E \rightarrow I}>0\right.$, light green) has a higher frequency than the pure 1180 ING rhythm $\left(\varepsilon_{E \rightarrow I}=0\right.$, blue line $)$ since the nonzero excitatory 1181 input from the $\mathrm{E}$ neuron advances the spiking of the I neuron. 1182 The higher the $\mathrm{E}$ drive, the earlier does the $\mathrm{E}$ spike arrive in the ${ }_{1183}$ period of the I neuron and the smaller is its excitatory effect 1184 due to the I neuron's PRC and transfer function [Fig. 2(c)]. 1185 The frequency of the ING rhythm thus slightly decreases with 1186 increasing E drive.

1187 The absence of a PING rhythm for small E drive, where the ${ }_{1188}$ pure ING frequency is higher than the pure PING frequency, 1189 can be understood from Eqs. (95) and (57), which specify 1190 the pure PING and pure ING frequencies, respectively. Equa1191 tion (95) implies that the pure PING frequency is determined by the interval between spikes of the E neuron, which is subject 1192 to the inhibition $\varepsilon_{I \rightarrow E}$ arriving at $\mathrm{E}$ phase $2 \tau$. According to 1193 Eq. (57), the pure ING frequency is determined by the interval ${ }_{1194}$ between spikes of the I neuron subject to the inhibition $\varepsilon_{I \rightarrow I}$. ${ }_{1195}$ In a full network generating PING, the inhibition arrives at ${ }_{1196}$ E phase $2 \tau$ or later, if the excitation of the I neuron is not ${ }_{1197}$ suprathreshold. Since the delaying effect of the inhibition 1198 increases the larger the E phase is at its arrival, the spiking 1199 interval of the full network E neuron is larger or equal to that ${ }_{1200}$ in the pure PING network. For the full network to generate ${ }_{1201}$ PING, the spiking interval of the E neuron subject to inhibition 1202 $\varepsilon_{I \rightarrow E}$ must at least be shorter than the spiking interval of the ${ }_{1203}$ I neuron subject to inhibition $\varepsilon_{I \rightarrow I}$ (the spiking interval in the ${ }_{1204}$ pure ING network), since the additionally arriving excitation 1205 $\varepsilon_{E \rightarrow I}$ further decreases the spike interval of the I neuron. ${ }_{1206}$ When already the frequency of pure ING is higher than that of ${ }_{1207}$ pure PING, this necessary condition is violated and the PING ${ }_{1208}$ rhythm is excluded.

As the $\mathrm{E}$ drive increases, the pure PING frequency starts 1210 to exceed the pure ING frequency [in Fig. 7(c) near $1 / \Theta_{E}=1211$ 0.46] and the full network becomes able to generate a PING ${ }_{1212}$ rhythm. In the subsequent parameter region, the full network ${ }_{1213}$ can generate either PING or ING depending on the initial state 1214 of the neurons. As the E drive increases further, the ING rhythm ${ }_{1215}$ disappears [near $1 / \Theta_{E}=0.47$ in Fig. 7(c)]. This is because the ${ }_{1216}$ phase advance of the I neuron due to the E spike becomes too ${ }_{1217}$ small compared to the decreasing interval between spikes of ${ }_{1218}$ the E neuron [Fig. 7(c): the light green curve meets the dark ${ }_{1219}$ green one]. We note that the (negative) slope of the light green 1220 curve is smaller in absolute value than the (positive) slope of ${ }_{1221}$ the dark green curve. In other words, the PING frequency is 1222 more sensitive to a change of the $\mathrm{E}$ drive $1 / \Theta_{E}$ than the ING ${ }_{1223}$ frequency.

\section{Analysis of PING-ING interactions in networks with type I E and type II I neurons}

We will now analyze interactions between PING and ING ${ }_{1227}$ rhythms for networks with type I LIF E and type II sine I ${ }_{1228}$ neurons for varying I and E drives; see Fig. 8. As in Fig. 7, 1229 the blue surface or curves in Fig. 8 represent the frequency of ${ }_{1230}$ the pure ING rhythm, red stands for the pure PING rhythm, 1231 and green for full network rhythms. The frequency of the pure ${ }_{1232}$ ING rhythm is again given by Eq. (57). The pure PING rhythm ${ }_{1233}$ assumes spiking of the I neuron at time $\tau$ after spiking of the ${ }^{1234}$ E neuron. The frequency of the pure PING rhythm is thus ${ }_{1235}$ again given by Eq. (95). As mentioned above (Sec. III B), the ${ }_{1236}$ sine I neuron without an external constant drive cannot reach ${ }_{1237}$ the threshold for finite value of $\varepsilon_{E \rightarrow I}$; it can nevertheless get ${ }_{1238}$ close, such that the temporal distance between E and I spike ${ }_{1239}$ is approximately $\tau$. We need to keep this point in mind when 1240 comparing pure PING and full network PING.

In contrast to the case of networks with type I E and I 1242 neurons, the full network with type I E and type II I neurons ${ }_{1243}$ generates a stable oscillation with a frequency between those of ${ }_{1244}$ pure ING and pure PING rhythms. Furthermore, our analysis ${ }_{1245}$ reveals an unstable oscillation (scenario 2) generated by the 1246 full network, with a frequency that is much higher than the ${ }_{1247}$ stable one for our parameters. For smaller I drive [lower 1248 $1 / \Theta_{I}$; see Fig. 8(b)] the full network generates a PING rhythm 1249 
(a)

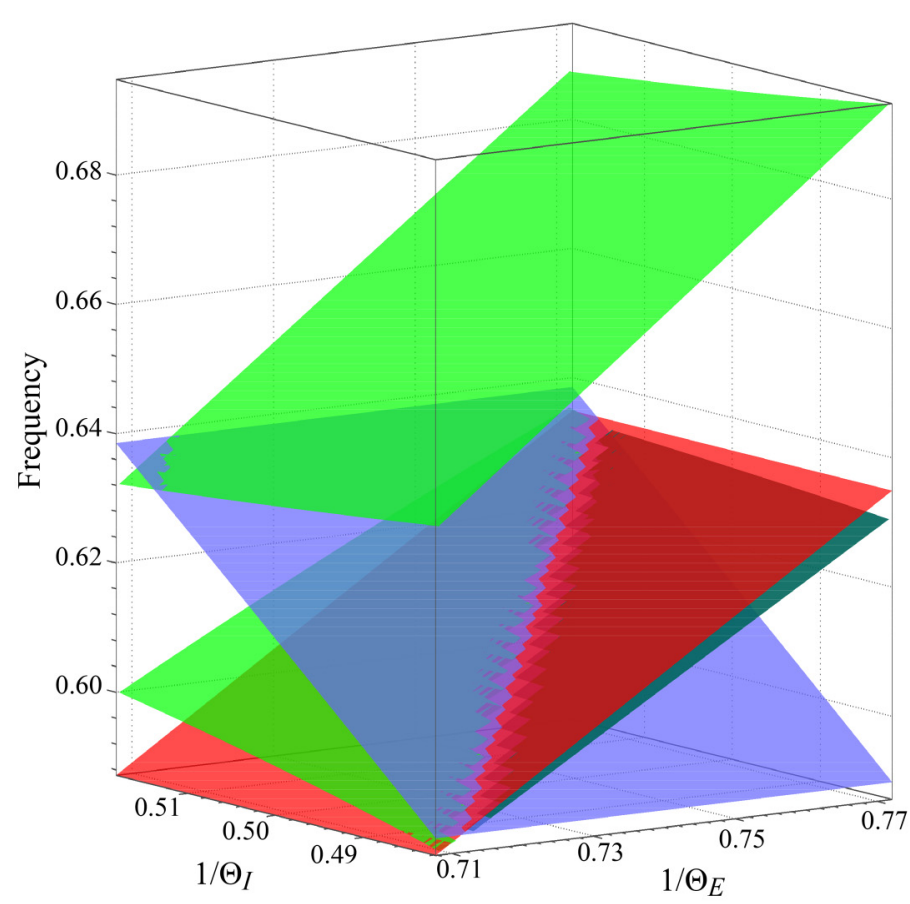

(b)

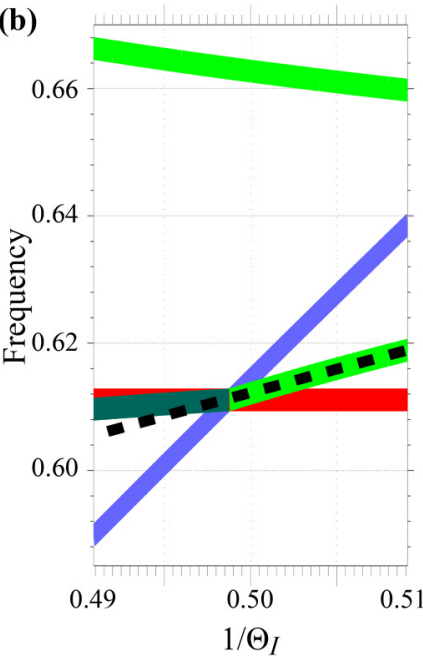

(c)

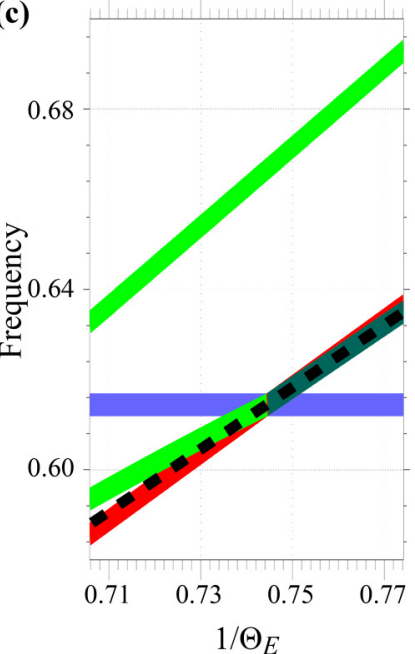

FIG. 8. Transitions between PING and ING in a network of a type I (leaky integrate-and-fire) E neuron and a type II (sine) I neuron. The blue and red surfaces or curves show the oscillation frequencies of pure ING and pure PING rhythms, respectively. The green surfaces or curves show the frequency of oscillations in the full two-neuron network. Panel (a) displays the frequency of network oscillations versus the E and I drives (measured by intrinsic period ${ }^{-1}$ ). Panels (b) and (c) show cross sections of the surfaces given in (a): The drive of the I neuron (b) or of the E neuron (c) increases from left to right while the other drive is kept fixed. The light green (b) or the dark green (c) curves are continued by black dashed lines with the curves' average slope to allow a better comparison to the slopes of the other curves. The light green surface with comparably high frequencies in (a) and the related light green curves in (b) and (c) correspond to a scenario 2 unstable ING rhythm, while the light green surface and curves with lower frequency correspond to a scenario 3 stable ING rhythm. Dark green shows the frequency of the full network PING rhythm (scenarios 5, 1 in alternation). Parameter settings: $\varepsilon_{I \rightarrow E}=-0.2, \varepsilon_{E \rightarrow I}=0.5, \varepsilon_{I \rightarrow I}=-0.42$, and $\tau=0.4$; in (b) the drive to the E neuron is $1 / \Theta_{E}=0.74$ and in (c) the drive to the I neuron is $1 / \Theta_{I}=0.5$.

1250 [alternating scenarios 5 and 1; dark green curve in Fig. 8(b)]. 1251 Its frequency is higher than the pure ING frequency; this is 1252 due to the fact that in the PING rhythm the E spike arrives 1253 in the second part of period of the sine neuron, i.e., between $1254] \Theta_{I} / 2, \Theta_{I}[$, and it thus has an excitatory effect. Since the E 1255 spike brings the I neuron only close to its threshold $\Theta_{I}$, the 1256 next spike time still depends on the I drive: The larger the 1257 drive, the shorter the time that the I neuron needs to reach the 1258 threshold after the E-spike arrival. Since this time is always at 1259 least slightly larger than zero, the full network PING frequency 1260 is lower than the pure PING frequency.

1261 As we increase the I drive further, the full network switches 1262 from operating in PING mode to ING mode [scenario 3; light 1263 green curve in Fig. 8(b); the switch occurs near $\left.1 / \Theta_{I}=0.5\right]$.
As for networks of two type I neurons [cf. Fig. 7(b)], the 1264 rate of change of ING frequency is higher than that of PING ${ }_{1265}$ frequency; the ING frequency is more sensitive to a change 1266 of the I drive $1 / \Theta_{I}$ than the PING frequency [compare the ${ }_{1267}$ dark green curve with the black dashed line in Fig. 8(b)]. The ${ }_{1268}$ ING rhythm [light green curve in Fig. 8(b)] appears, in contrast ${ }_{1269}$ to the case of two type I neurons, at the same point where the 1270 PING rhythm vanishes. The latter happens where the frequency 1271 of the pure ING rhythm becomes higher than that of the pure 1272 PING rhythm. This can be understood as in the case of two 1273 type I neurons, since the excitatory input in the full network 1274 PING also advances the phase of the type II I neuron. The full 1275 network ING frequency is smaller than the pure ING frequency 1276 because in the full network there is an additional input from the ${ }_{1277}$ 

1318 impact.

1331 dal cells in different brain areas belong to the category of type 1332 I neurons [73-75] (see, however, [76-78]). We adopt this view ${ }_{1333}$ and model the E neuron as a (type I) leaky integrate-and-fire 1334 neuron. We review the derivation of the phase representation 1335 for this model, in particular, the derivation of the transfer function $H$, which maps the phase of the neuronal oscillator ${ }_{1336}$ before synaptic input to the phase after synaptic input. A full, ${ }_{1337}$ general derivation of the phase representation for type I neurons ${ }_{1338}$ was provided in a previous study (see [65]). The appropriate ${ }_{1339}$ choice of interneuron phase response curve type is less clear. ${ }_{1340}$ Oscillation-relevant interneurons can be either of type I [79] ${ }_{1341}$ or type II [62] depending on the brain area. Therefore, we ${ }_{1342}$ consider both options in our study: We model the I neuron as ${ }_{1343}$ a type I leaky integrate-and-fire neuron or as a type II sine ${ }_{1344}$ neuron. The interactions between the neurons are modeled by ${ }_{1345}$ Dirac delta pulses, which induce a jump in the voltage of the ${ }_{1346}$ receiving neuron by an amount that is described by the strength ${ }_{1347}$ of the synaptic connection and independently of the voltage. In ${ }_{1348}$ the present study we show how to derive the phase dynamics 1349 for such neural oscillators, if they have an iPRC of type II. In ${ }_{1350}$ particular, for our type II sine I neuron, we derive the voltage ${ }_{1351}$ dynamics and the full phase representation from its iPRC. ${ }_{1352}$ The chosen iPRC shows a change from negative to positive as 1353 typical for type II neurons. Concretely, we use the (inverted) ${ }_{1354}$ sine iPRC of a normal form oscillator of the Hopf bifurcation 1355 (cf. [68]). Using the phase description we can provide a full 1356 theoretical analysis of the dynamics of a network model with ${ }_{1357}$ an E neuron and an I neuron of arbitrary type and arbitrary ${ }_{1358}$ details of the dynamics.

Our results are also relevant for single oscillator studies, 1360 since they allow us to investigate how different an oscillator ${ }_{1361}$ model is from a model expressible by one-dimensional voltage ${ }_{1362}$ dynamics with voltage-independent inputs. As an example, ${ }_{1363}$ we consider the classical radial isochron clock $[1,5,80]$. In ${ }_{1364}$ this model, a point circulates on its attractor cycle in the 1365 $x, y$ plane. Synaptic inputs cause deviations from the stable ${ }_{1366}$ attractor cycle. Assuming that the radial deflection after an ${ }_{1367}$ input quickly relaxes back while the change in the angular ${ }_{1368}$ variable remains, this model reduces to a phase oscillator. For ${ }_{1369}$ infinitesimal inputs, the resulting phase response is given by 1370 a sine iPRC. However, comparing the PRC with that in our 1371 study reveals a difference in the series expansion of the synaptic ${ }_{1372}$ strength $\varepsilon$ from second order on; see the Appendix. $\quad{ }_{1373}$

To theoretically investigate oscillations in our two-neuron ${ }_{1374}$ networks, we first provide a basic framework by deriving the ${ }_{1375}$ five relevant scenarios for the change of phase differences upon 1376 interactions of the $\mathrm{E}$ and I neurons (see Fig. 4). This allows us 1377 to construct various modes of synchronization [71] between ${ }_{1378}$ the two oscillators by concatenating and repeating scenarios 1379 and determining whether this results in periodic dynamics. For 1380 example, scenarios 5 and 1 can be concatenated in alternation ${ }_{1381}$ to obtain 1:1 synchronization between the $\mathrm{E}$ and I oscillators. ${ }_{1382}$ For our study, we focus on 1:1 synchronization because both ${ }_{1383}$ the population of interneurons and the population of pyramidal ${ }_{1384}$ cells display increased activity only once per gamma cycle ${ }_{1385}$ $[81,82]$.

When our two-neuron network operates in PING mode, the ${ }_{1387}$ output of the E neuron elicits the spiking of the I neuron. ${ }_{1388}$ This happens in scenario 4 and it can happen in the mode ${ }_{1389}$ of alternating scenarios 5 and 1 . The interpretation of a mode 1390 with repeating scenario 4 as PING is straightforward, due to 1391 the suprathreshold excitation of the I neuron. In contrast, the ${ }_{1392}$ interpretation of modes of alternating scenarios 5,1 requires 1393 some caution. Such modes should be interpreted as PING, 1394 if the E neuron nearly excites the I neuron to spike, i.e., if ${ }_{1395}$ 
the E neuron's spike brings the I neuron so close to threshold that it spikes shortly thereafter. In the considered parameter region around the crossing of the pure PING and the pure ING network oscillation frequencies, this is the case in all our simulations of scenarios 5,1 rhythms: The I neuron spikes less than $0.1 T$ after the $\mathrm{E}$ spike arrives, where $T$ is the period of the rhythm. For simplicity, we therefore refer to the scenarios 5,1 rhythm as PING throughout the present article. A comparison with experimental findings corroborates our interpretation: Ref. [83] demonstrates that in PING the discharge probability of the CA3 pyramidal cells in the gamma cycle $(T \approx 18.9 \mathrm{~ms}$ ) reaches its maximum $3.1 \mathrm{~ms}$ before the maximal discharge probability of the CA3 interneurons. The latency of a monosynaptic connection is approximately 1.3 $\mathrm{ms}[84,101]$, so the discharge probability of the interneurons reaches a maximum $1.8 \mathrm{~ms}(=3.1 \mathrm{~ms}-1.3 \mathrm{~ms})$ after the arrival of the inputs. This temporal difference is about $(1.8 / 18.9) \approx$ 0.1 of the oscillation period $T$.

We find that when the full network operates in PING mode, its frequency is more sensitive to changes of the external drive to the E neuron than to changes of the external drive to the I neuron [see Fig. 7, panels (b) and (c), and Fig. 8, panels (b) and (c)]. When the full network operates in ING mode, the frequency more strongly depends on the external current given to the I neuron.

Our theoretical study also shows that the qualitative relation of the frequency of the full network and the frequencies of pure ING oscillations $\left(\varepsilon_{E \rightarrow I}=0\right)$ and of pure PING oscillations (no or negligible I drive) depends on whether the I neuron belongs to the category of type I or type II. When the I neuron is a type I LIF neuron, the frequency of the full network is above the pure ING and pure PING frequencies or equals the pure PING frequency. The former can be understood from the fact that the excitatory output from the E neuron to the I neuron advances the phase of the type I I neuron and therefore shortens the cycle and increases the frequency. In contrast, when the I neuron is a type II sine neuron, the frequency of the full network is between the frequencies of pure ING and pure PING. This can be understood from the fact that the excitatory input from the E neuron delays the phase of the I neuron when the spike from the E neuron arrives early in the phase of the I neuron. This increases the cycle duration and thus decreases the frequency.

Throughout the article, the type I neurons in our networks are LIF neurons. We have likewise explored networks with two type I quadratic integrate-and-fire (QIF) neurons [5] in phase representation (cf. Sec. II). In these networks with the QIF E neuron and QIF I neuron, we observe the same qualitative frequency relations as in networks of two LIF neurons, if the pure ING frequency is higher than the pure PING frequency: The frequency of the full network is slightly above the pure ING frequency. However, when the pure PING frequency is higher than the pure ING frequency, the full network frequency of coupled QIF neurons is below the pure PING frequency. This is because in the pure PING rhythm we assume that the excitatory input excites the I neuron to spike immediately at its arrival. For a QIF I neuron, this would require an infinitely large excitatory coupling strength. Since in the full network the coupling strengths are finite, the QIF I neuron cannot reach threshold instantaneously at spike arrival, in contrast to a LIF neuron. Consequently, the QIF I neuron spikes later in the cycle and the full network frequency is lower than the pure ${ }_{1456}$ PING frequency.

When we compare the results of the two-neuron networks, 1458 which contain two LIF or one LIF and one sine neuron, to 1459 the results from simulations in a large network of biologically ${ }_{1460}$ more detailed pyramidal cells and interneurons, the latter show ${ }_{1461}$ similar qualitative relations [58]: The frequency of the full 1462 network with type I interneurons is slightly above the frequency ${ }_{1463}$ of pure ING and of pure PING, while the frequency of the full ${ }_{1464}$ network with type II interneurons can be in between. However, 1465 the full network PING frequency of the two-neuron network ${ }_{1466}$ with the type II I neuron is intermediate between the pure ING ${ }_{1467}$ and pure PING frequencies [cf. Fig. 8, panels (b) and (c)], while ${ }_{1468}$ it is slightly above for the large networks (cf. Fig. 7, panels (b) 1469 and (c), in Ref. [58]). The key to understanding this discrepancy 1470 is the net value of the excitatory output from the E neuron (or ${ }_{1471}$ from the population of the pyramidal cells) to the I neuron ${ }_{1472}$ (or to the population of the interneurons). In the pure PING ${ }_{1473}$ two-neuron network the coupling is assumed to be so strong ${ }^{1474}$ that the E spike excites the I neuron to spike immediately, 1475 while in the full two-neuron network the I neuron's phase ${ }_{1476}$ still needs to slightly increase to reach threshold. This causes 1477 the frequency of pure PING to be higher than that of the full ${ }_{1478}$ network. However, the net values of the excitatory outputs in 1479 both large-network topologies are approximately the same. ${ }_{1480}$ With additional drive to the interneurons in the full large ${ }_{1481}$ network, its frequency is thus higher than that of the pure PING ${ }_{1482}$ large network. Another discrepancy between the results for ${ }_{1483}$ the two-neuron network and the results for the large networks ${ }_{1484}$ in Ref. [58] concerns network bistability. The phase iteration ${ }_{1485}$ map of two-neuron networks with type I LIF E and I neurons ${ }_{1486}$ has two stable fixed points (one corresponding to ING and one ${ }_{1487}$ corresponding to PING) for parameter values near the crossing ${ }_{1488}$ of the pure ING and pure PING frequencies, giving rise to 1489 bistability between ING and PING; see Fig. 7, panels (b) and 1490 (c). In contrast, the simulations of the large network reveal ${ }_{1491}$ only one oscillation frequency near the crossing. Presumably, 1492 this is due to noise added to the input to the neurons in 1493 the large network. This gives rise to slightly different firing ${ }_{1494}$ frequencies of the network's neurons, which may together 1495 obscure the bistability into a gradual transition between ING ${ }_{1496}$ and PING. A second fixed point also occurs for the phase ${ }_{1497}$ iteration map of the two-neuron network with the type II I ${ }_{1498}$ neuron; cf. Figs. 6 and 8. It is unstable and corresponds to 1499 an unstable oscillation with higher frequency. In contrast, the 1500 large network simulations again reveal only one frequency. An ${ }_{1501}$ obvious explanation is that the employed simulations cannot 1502 generate unstable oscillations due to noise. Although the results $\quad{ }_{1503}$ based on the two-neuron networks and the large networks 1504 [58] yield differences in some detail, the general picture is 1505 similar. In particular, the stable rhythm of the full network is 1506 usually realized by the one of ING or PING that generates ${ }_{1507}$ the higher frequency. That is, the mechanism that generates ${ }_{1508}$ the higher frequency "wins" in the sense that it determines the ${ }_{1509}$ frequency of the full network. In the two-neuron network this 1510 is also the rhythm that generates the higher frequency in the ${ }_{1511}$ corresponding pure networks. The rough explanation is that the 1512 higher frequency generating mechanism absorbs the resources $\quad 1513$ necessary to maintain a rhythm: A neuron will generally spike ${ }_{1514}$ earlier due to recruitment into a higher frequency rhythm and is 1515 
1516 then not able to spike again to contribute to the lower frequency 1517 one. However, our analytical approaches in the present article 1518 allow for more detailed analyses; see Sec. VI.

1519 Most studies with a large impact on the field using two1520 neuron (oscillator) networks were conducted either for purely 1521 inhibitory networks [85-92] or purely excitatory networks 1522 [85,90,92-96]. Studies for two-neuron networks, in which 1523 one is excitatory and another is inhibitory, are less common 1524 and many of them are in different contexts [42,74,97-99].

1525 Börgers and Kopell [56] presented a study related to ours, but 1526 without coupling delays and assuming that $\varepsilon_{E \rightarrow I}$ is always 1527 suprathreshold. The article reports that when the intrinsic 1528 frequency of the I neuron is higher than the frequency of 1529 the PING network rhythm, the latter is destroyed via phase 1530 walk-through, which results in an irregular oscillation (the I 1531 neuron spikes more than once per cycle).

1532 Our study considers both type I and type II I oscillators 1533 as well as a finite coupling delay. The consideration of 1534 the frequency aspect yields an intriguing dependence of the 1535 frequency changes when changing external drive, on the phase 1536 response curve of the oscillators as presented in Sec. VI.

1537 Unlike other methods for studying the two-neuron network, 1538 our method does not focus on determining the mode of the 1539 phase locking directly but based on fundamental interaction 1540 scenarios, which can be used to construct different modes of 1541 locking under the assumption that the phase difference between 1542 the two oscillators changes only when either an input arrives 1543 or a phase is reset; the assumption is valid in our study because 1544 the connections are modeled by Dirac delta pulses. By this, we 1545 consider fast postsynaptic current (PSC) kinetics that ignores 1546 a PSC's rise and decay. Van Vreeswijk et al. [85] and others 1547 [100] have shown that the duration of the PSCs relative to the 1548 interval of spiking is important. Since the time constant of the 1549 synapses relevant to gamma oscillations is on the order of a few 1550 milliseconds [52,101-103], which is short against the period 1551 of gamma oscillations (around $20 \mathrm{~ms}$ ), modeling the PSCs as 1552 delta pulses seems reasonable. The assumption that the choice 1553 of Dirac delta pulses does not affect the central conclusions 1554 of our study is also corroborated by our comparisons with 1555 biologically more detailed, larger scale networks [58].

1556 The results of this study are relevant for in vitro and in 1557 vivo experimental studies, since they imply that a seemingly 1558 straightforward interpretation of an observed rhythm as ING 1559 or PING has to be done with care. Our findings highlight 1560 that frequent firing of the pyramidal cells does not necessarily 1561 imply that the network is dominated by PING. Similar spike 1562 patterns can be generated both by ING and by PING rhythms. 1563 In particular, the network can generate ING rhythms, where 1564 the pyramidal cells spike before the interneurons (scenario 3).

1565 Various experiments show shifts of the frequency generated 1566 by cortical circuits when the influence of the excitatory input 1567 on the interneurons decreases due to optogenetic silencing of 1568 the local pyramidal cells in vivo [104] or applying an antagonist 1569 of fast excitatory synaptic coupling in vitro [105]. One might 1570 guess that if the cortical circuits produce oscillations whose 1571 frequency changes when one decreases the local excitatory 1572 input, the oscillations are likely to be PING because the 1573 oscillations depend on the excitation-inhibition loop. However, 1574 our studies in the two-neuron networks and in larger networks 1575 [58] suggest that knowing only that the frequency changes when removing the local $\mathrm{E}$ to I inputs $\varepsilon_{E \rightarrow I}$ (by silencing 1576 pyramidal cells or disabling fast excitatory synaptic inputs) is 1577 not enough to determine whether the cortical circuits operate 1578 in either PING or ING mode. We also need to know the type of 1579 the interneurons and the direction of change of the frequency 1580 to gain information about the operation mode. 1581

Overall, we provide a mathematical framework to construct $\quad{ }_{1582}$ phase oscillators that can be described by a single voltage 1583 variable with voltage-independent input, based on basically 1584 any smooth infinitesimal phase response curve. Furthermore, 1585 we construct iteration maps characterizing the dynamics of 1586 two-neuron networks. We use them to analyze how regular ${ }_{1587}$ PING and ING oscillations in the two-neuron networks inter- 1588 act. Our results show that the winning mechanism (either PING ${ }_{1589}$ or ING) is the one with the higher frequency in the full and 1590 pure networks. Except for possible small coexistence regions 1591 it will suppress the other one since it absorbs all "resources" 1592 (neurons ready to spike) available to maintain a rhythm. 1593

\section{ACKNOWLEDGMENTS}

1594

We thank P. Tiesinga, F. Battaglia, and S. Jahnke for ${ }_{1595}$ fruitful discussions. The project was supported by Nederlandse 1596 Organisatie voor Wetenschappelijk Onderzoek (NWO 11051597 051.02.050), by the Max Kade Foundation New York, and 1598 by Bundesministerium für Bildung und Forschung (BMBF) 1599 through the Bernstein Network (Bernstein Award 2014). $\quad 1600$

\section{APPENDIX: COMPARISON OF OUR SINE NEURON WITH 160 THE RADIAL ISOCHRON CLOCK

The radial isochron clock (RIC) or Andronov-Hopf oscilla- ${ }_{1603}$ tor (e.g., $[1,5,80]$ ) is the normal form of oscillating systems near ${ }_{1604}$ Hopf bifurcations. It is a two-dimensional dynamical system 1605 with the unit cycle as attractor. The dynamical equations for ${ }_{1606}$ the radial and angular state variables are

$$
\begin{gathered}
\frac{d r}{d t}=\Lambda r\left(1-r^{2}\right), \\
\frac{d \varphi}{d t}=1
\end{gathered}
$$

with sufficiently large parameter $\Lambda$ such that deflections in the ${ }_{1608}$ radial direction are quickly eliminated and input pulses meet 1609 the system practically on the limit cycle. In contrast, angular 1610 perturbations remain; see Eq. (A2). The oscillator spikes and 1611 is reset when its angle reaches $\Theta=2 \pi$ from below. One can 1612 now posit that inputs cause a deflection into the direction of 1613 the $x$ coordinate,

$$
\left[\begin{array}{c}
\cos (\varphi) \\
\sin (\varphi)
\end{array}\right] \rightarrow\left[\begin{array}{c}
\cos (\varphi)+\varepsilon \\
\sin (\varphi)
\end{array}\right]
$$

see $[5,80]$. Note that by this definition an input cannot cause 1615 the oscillator to cross threshold, as it changes the state parallel 1616 to it. Assuming that we are and stay in the first quadrant, 1617 the angle changes as $\varphi \rightarrow \arctan \left(\frac{\sin (\varphi)}{\varepsilon+\cos (\varphi)}\right)$. Since the angular 1618 deflection is conserved while the radial variable relaxes to one, 1619 the phase after the input is $H_{\mathrm{RIC}}(\varphi, \varepsilon)=\arctan \left(\frac{\sin (\varphi)}{\varepsilon+\cos (\varphi)}\right)$. If ${ }_{1620}$ we do not stay within the first quadrant, we need to extend the 1621 
definition,

$$
H_{\mathrm{RIC}}(\varphi, \varepsilon)= \begin{cases}\arctan \left(\frac{\sin (\varphi)}{\varepsilon+\cos (\varphi)}\right), & \text { for } \varphi \in] 0, \pi[\text { and } \cos (\varphi)+\varepsilon>0, \\ \arctan \left(\frac{\sin (\varphi)}{\varepsilon+\cos (\varphi)}\right)+\pi, & \text { for } \cos (\varphi)+\varepsilon<0, \\ \arctan \left(\frac{\sin (\varphi)}{\varepsilon+\cos (\varphi)}\right)+2 \pi, & \text { for } \varphi \in] \pi, 2 \pi[\text { and } \cos (\varphi)+\varepsilon>0,\end{cases}
$$

1623 with the appropriate continuations at the borders. The first 1624 derivative with respect to $\varepsilon$ reads

$$
\frac{\partial H_{\mathrm{RIC}}(\varphi, \varepsilon)}{\partial \varepsilon}=-\frac{\sin (\varphi)}{1+2 \varepsilon \cos (\varphi)+\varepsilon^{2}} .
$$

1625 Equation (A5) specifies in linear approximation the change of 1626 the current phase $H_{\mathrm{RIC}}(\varphi, \varepsilon)$, in terms of the already received 1627 input $\varepsilon$ and the initial phase $\varphi$. This is conceptually related to 1628 Eq. (23). It is distinct from a differential equation for the current 1629 phase, which specifies the change of the current phase in terms 1630 of the current phase [like Eq. (31)] and, if nonautonomous (see 1631 below), the independent variable, i.e., where the right-hand side 1632 would be a function of $H_{\mathrm{RIC}}(\varphi, \varepsilon)$ and $\varepsilon$. For $\varepsilon=0$ Eq. (A5) 1633 yields the iPRC. Since

$$
\left.\frac{\partial H_{\mathrm{RIC}}(\varphi, \varepsilon)}{\partial \varepsilon}\right|_{\varepsilon=0}=-\sin (\varphi),
$$

1634 the neuron is a sine neuron. It is, however, not the same sine 1635 neuron as ours; see Sec. IIIB. The transfer function of our 1636 sine neuron can be obtained via the autonomous differential 1637 equation

$$
\frac{\partial H_{\text {sine }}(\varphi, \varepsilon)}{\partial \varepsilon}=Z\left(H_{\text {sine }}(\varphi, \varepsilon)\right)=-\sin \left[H_{\text {sine }}(\varphi, \varepsilon)\right],
$$

(a)

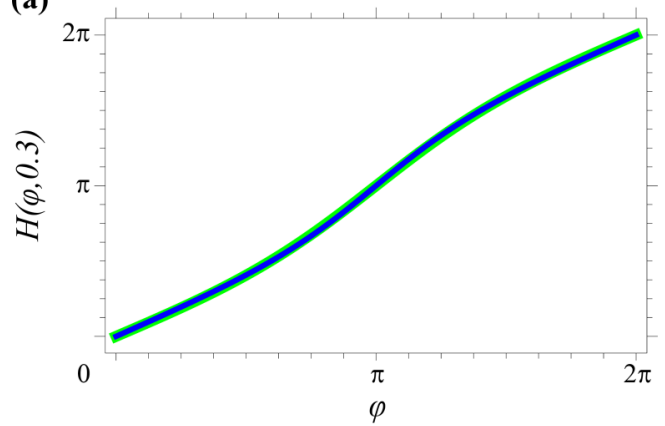

(c)

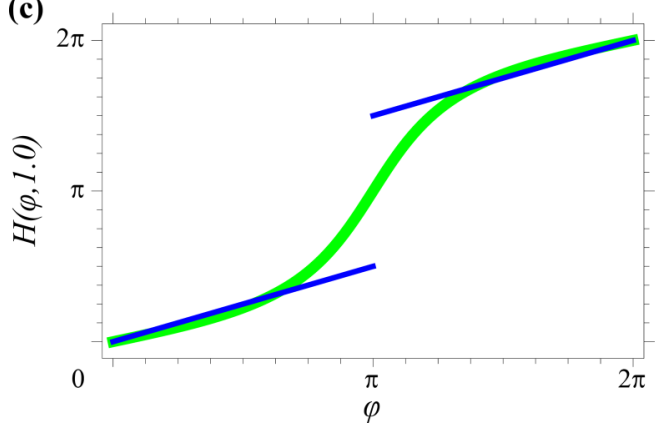

with initial condition $H_{\text {sine }}(\varphi, 0)=\varphi$; cf. Eq. (31). The righthand side of the equation does not depend on $\varepsilon$ and is therefore uniquely specified by the iPRC. Solving Eq. (A7) using separation of variables yields for a neuron with period $\Theta=2 \pi$

$$
\begin{aligned}
& H_{\text {sine }}(\varphi, \varepsilon) \\
& \quad= \begin{cases}2 \arctan \left[\tan \left(\frac{\varphi}{2}\right) e^{-\varepsilon}\right], & \text { for } \varphi \in] 0, \pi[, \\
2 \arctan \left[\tan \left(\frac{\varphi}{2}\right) e^{-\varepsilon}\right]+2 \pi, & \text { for } \varphi \in] \pi, 2 \pi[,\end{cases}
\end{aligned}
$$

with appropriate continuations; $\mathrm{cf}$. Eq. (39). The first derivative [e.g., computed from Eq. (A7)] then explicitly reads

$$
\begin{aligned}
\frac{\partial H_{\text {sine }}(\varphi, \varepsilon)}{\partial \varepsilon} & =-\sin \left[H_{\text {sine }}(\varphi, \varepsilon)\right] \\
& =-\sin \left\{2 \arctan \left[\tan \left(\frac{\varphi}{2}\right) e^{-\varepsilon}\right]\right\} \\
& =-\frac{2 e^{\varepsilon} \tan \left(\frac{\varphi}{2}\right)}{e^{2 \varepsilon}+\tan \left(\frac{\varphi}{2}\right)^{2}}
\end{aligned}
$$

which agrees only for $\varepsilon=0$ with Eq. (A5). We may conclude $\quad 1645$ that $H_{\mathrm{RIC}}(\varphi, \varepsilon)$ does not obey the autonomous differential ${ }_{1646}$

(b)

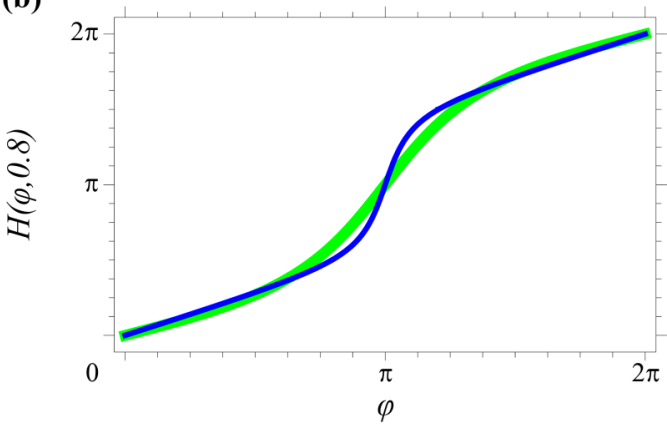

(d)

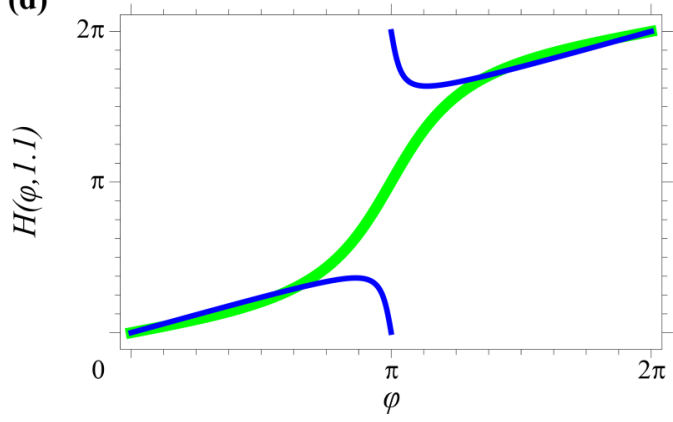

FIG. 9. Comparison of $H_{\text {sine }}(\varphi, \varepsilon)$ (green) with $H_{\text {RIC }}(\varphi, \varepsilon)$ (blue) for different values of $\varepsilon$. Panels (a), (b), (c), and (d) show the transfer functions for $\varepsilon=0.3,0.8,1$, and 1.1 , respectively. 
1647 equation Eq. (31), but a nonautonomous one, where the right1648 hand side depends explicitly on the independent variable $\varepsilon$ 1649 and which reduces to the iPRC at $\varepsilon=0$. Graphically speak1650 ing, consider a small input piece $d \tilde{\varepsilon}$ of a total input $\varepsilon$. $d \tilde{\varepsilon}$ 1651 arrives after the input piece $\tilde{\varepsilon}$ of $\varepsilon$ has already been received. 1652 Then the impact of $d \tilde{\varepsilon}$ does not only depend on the phase ${ }_{1653} \varphi(\tilde{\varepsilon})=H_{\mathrm{RIC}}(\varphi, \tilde{\varepsilon})$ reached due to $\tilde{\varepsilon}$ but also explicitly on $\tilde{\varepsilon}$ 1654 itself.

1655 The series expansions in $\varepsilon$ of $H_{\mathrm{RIC}}(\varphi, \varepsilon)$ and $H_{\text {sine }}(\varphi, \varepsilon)$ 1656 around zero differ from second order on (they agree by 1657 definition up to first order),

$$
\begin{aligned}
H_{\mathrm{RIC}}(\varphi, \varepsilon)= & \varphi-\sin (\varphi) \varepsilon+\frac{1}{2} \sin (2 \varphi) \varepsilon^{2} \\
& -\frac{1}{3} \sin (3 \varphi) \varepsilon^{3}+O\left(\varepsilon^{4}\right),
\end{aligned}
$$

$$
\begin{aligned}
H_{\text {sine }}(\varphi, \varepsilon)= & \varphi-\sin (\varphi) \varepsilon+\frac{1}{4} \sin (2 \varphi) \varepsilon^{2} \\
& -\frac{1}{12}[\sin (3 \varphi)-\sin (\varphi)] \varepsilon^{3}+O\left(\varepsilon^{4}\right) .
\end{aligned}
$$

Equations (31) and (A7) allow us to compute expressions for 1658 the higher order derivatives and thus Taylor coefficients of its 1659 solution by differentiating both sides and replacing derivatives 1660 appearing on the right-hand side using the original equa- 1661 tion. We note that as second derivative we obtain $\frac{\partial^{2} H(\varphi, \varepsilon)}{\partial \varepsilon^{2}}={ }_{1662}$ $Z^{\prime}(\varphi) Z(\varphi)$, which implies a second order Taylor coefficient ${ }_{1663}$ $\frac{1}{2}[\sin (\varphi) \cos (\varphi)]=\frac{1}{4} \sin (2 \varphi)$ as present in Eq. (A11) but not 1664 in Eq. (A10). Figure 9 illustrates the increasing discrepancy 1665 of $H_{\mathrm{RIC}}(\varphi, \varepsilon)$ and $H_{\text {sine }}(\varphi, \varepsilon)$ for increasing $\varepsilon$. For $\varepsilon=1,1666$ $H_{\mathrm{RIC}}(\varphi, \varepsilon)$ has a singularity (at $\left.\varphi=\pi\right)$ and beyond a discon- 1667 tinuity.
[1] E. Izhikevich, Dynamical Systems in Neuroscience: The Geometry of Excitability and Bursting (MIT Press, Cambridge, 2007).

[2] A. T. Winfree, J. Theor. Biol. 16, 15 (1967).

[3] R. M. Smeal, G. B. Ermentrout, and J. A. White, Philos. Trans. R. Soc. London B 365, 2407 (2010).

[4] R. Mirollo and S. Strogatz, SIAM J. Appl. Math. 50, 1645 (1990).

[5] P. Goel and B. Ermentrout, Phys. D (Amsterdam, Neth.) 163, 191 (2002).

[6] A. Viriyopase, I. Bojak, M. Zeitler, and S. Gielen, Front. Comput. Neurosci. 6, 49 (2012).

[7] S. Jahnke, R.-M. Memmesheimer, and M. Timme, Phys. Rev. Lett. 100, 048102 (2008).

[8] R.-M. Memmesheimer and M. Timme, Phys. Rev. Lett. 97, 188101 (2006).

[9] J. Nishimura and E. J. Friedman, Phys. Rev. Lett. 106, 194101 (2011).

[10] G. Brandner, U. Schilcher, and C. Bettstetter, Comput. Networks 97, 74 (2016).

[11] T. Forrest, J. Ariaratnam, and S. H. Strogatz, J. Acoust. Soc. Am. 103, 2827 (1998)

[12] R. Goebel, R. R. Sanfelice, and A. R. Teel, Hybrid Dynamical Systems: Modeling, Stability, and Robustness (Princeton University Press, Princeton, 2012).

[13] L. Lapicque, J. Physiol. Pathol. Gén. 9, 620 (1907).

[14] W. M. Kistler, W. Gerstner, and J. L. v. Hemmen, Neural Comput. 9, 1015 (1997)

[15] L. Abbott and T. B. Kepler, in Proceedings of the XIth Sitges Conference on Statistical Mechanics of Neural Networks, Barcelona, 1990, edited by L. Garrido (Springer-Verlag, Berlin, 1990), p. 5.

[16] P. Dayan and L. Abbott, Theoretical Neuroscience: Computational and Mathematical Modeling of Neural Systems (MIT Press, Cambridge, 2001).

[17] G. Buzsáki, Rhythms of the Brain (Oxford University Press, Oxford, 2006).

[18] G. Thut, C. Miniussi, and J. Gross, Curr. Biol. 22, R658 (2012).

[19] G. Buzsáki and J. J. Chrobak, Curr. Opin. Neurobiol. 5, 504 (1995).

[20] G. Buzsáki, Z. Horváth, R. Urioste, J. Hetke, and K. Wise, Science 256, 1025 (1992).
[21] J. O'Keefe and M. L. Recce, Hippocampus 3, 317 (1993).

[22] A. Luczak, B. L. McNaughton, and K. D. Harris, Nat. Rev. Neurosci. 16, 745 (2015).

[23] E. Arabzadeh, S. Panzeri, and M. E. Diamond, J. Neurosci. 26, 9216 (2006).

[24] L. Wang, R. Narayan, G. Graña, M. Shamir, and K. Sen, J. Neurosci. 27, 582 (2007).

[25] N. Caporale and Y. Dan, Annu. Rev. Neurosci. 31, 25 (2008).

[26] R. Azouz and C. M. Gray, Neuron 37, 513 (2003).

[27] M. Volgushev, M. Chistiakova, and W. Singer, Neuroscience 83, 15 (1998).

[28] J. Jacobs, M. J. Kahana, A. D. Ekstrom, and I. Fried, J. Neurosci. 27, 3839 (2007).

[29] P. Fries, Neuron 88, 220 (2015).

[30] P. Fries, Trends Cogn. Sci. 9, 474 (2005).

[31] T. Womelsdorf, J. M. Schoffelen, R. Oostenveld, W. Singer, R. Desimone, A. K. Engel, and P. Fries, Science 316, 1609 (2007).

[32] A. M. Bastos, J. Vezoli, and P. Fries, Curr. Opin. Neurobiol. 31, 173 (2015).

[33] S. Jahnke, R.-M. Memmesheimer, and M. Timme, Phys. Rev. E 89, 030701 (2014).

[34] S. Jahnke, R.-M. Memmesheimer, and M. Timme, PLoS Comput. Biol. 10, e1003940 (2014).

[35] W. Singer, Neuron 24, 49 (1999).

[36] C. Tallon-Baudry and O. Bertrand, Trends Cogn. Sci. 3, 151 (1999).

[37] R. Eckhorn, in The Self-Organizing Brain: From Growth Cones to Functional Networks, Progress in Brain Research, edited by H. U. J. Van Pelt, M. A. Corner, and F. L. D. Silvar (Elsevier Science, 1994), Vol. 102, p. 405.

[38] J. M. Palva, S. Palva, and K. Kaila, J. Neurosci. 25, 3962 (2005).

[39] W. Singer and C. M. Gray, Annu. Rev. Neurosci. 18, 555 (1995).

[40] J. E. Lisman and M. A. Idiart, Science 267, 1512 (1995).

[41] A. L. Giraud and D. Poeppel, Nat. Neurosci. 15, 511 (2012).

[42] C. Börgers and N. J. Kopell, Neural Comput. 20, 383 (2008).

[43] J. J. Hopfield, Nature (London) 376, 33 (1995).

[44] M. A. Whittington, M. O. Cunningham, F. E. LeBeau, C. Racca, and R. D. Traub, Dev. Neurobiol. 71, 92 (2011).

[45] G. Buzsáki and X. J. Wang, Annu. Rev. Neurosci. 35, 203 (2012).

[46] L. L. Colgin and E. I. Moser, Physiology 25, 319 (2010). 
[47] S. R. Cobb, E. H. Buhl, K. Halasy, O. Paulsen, and P. Somogyi, Nature (London) 378, 75 (1995).

[48] W. W. Lytton and T. J. Sejnowski, J. Neurophysiol. 66, 1059 (1991).

[49] R. D. Traub, M. A. Whittington, S. B. Colling, G. Buzsáki, and J. G. Jefferys, J. Physiol. 493, 471 (1996).

[50] M. A. Whittington, R. D. Traub, and J. G. Jefferys, Nature (London) 373, 612 (1995).

[51] M. Whittington, R. Traub, N. Kopell, B. Ermentrout, and E. Buhl, Int. J. Psychophysiol. 38, 315 (2000).

[52] M. Bartos, I. Vida, and P. Jonas, Nat. Rev. Neurosci. 8, 45 (2007).

[53] N. Kopell and B. Ermentrout, Proc. Natl. Acad. Sci. USA 101, 15482 (2004).

[54] C. Börgers and N. Kopell, Neural Comput. 15, 509 (2003).

[55] P. H. Tiesinga, J. M. Fellous, J. V. José, and T. J. Sejnowski, Hippocampus 11, 251 (2001).

[56] C. Börgers and N. Kopell, Neural Comput. 17, 557 (2005).

[57] C. Börgers and B. Walker, Front. Comput. Neurosci. 7, 10 (2013).

[58] A. Viriyopase, R.-M. Memmesheimer, and S. Gielen, J. Neurophysiol. 116, 232 (2016).

[59] D. Hansel, G. Mato, and C. Meunier, Neural Comput. 7, 307 (1995).

[60] A. Erisir, D. Lau, B. Rudy, and C. S. Leonard, J. Neurophysiol. 82, 2476 (1999).

[61] T. Tateno, A. Harsch, and H. Robinson, J. Neurophysiol. 92, 2283 (2004).

[62] T. Tateno and H. Robinson, Biophys. J. 92, 683 (2007).

[63] C. van Vreeswijk, Phys. Rev. E 54, 5522 (1996).

[64] M. Timme, F. Wolf, and T. Geisel, Chaos 13, 377 (2003).

[65] R.-M. Memmesheimer and M. Timme, Phys. D (Amsterdam, Neth.) 224, 182 (2006).

[66] A. L. Hodgkin and A. F. Huxley, J. Physiol. 117, 500 (1952).

[67] B. Ermentrout, Neural Comput. 8, 979 (1996).

[68] E. N. Brown, J. Moehlis, and P. Holmes, Neural Comput. 16, 673 (2004).

[69] W. Gerstner and W. M. Kistler, Spiking Neuron Models: Single Neurons, Populations, Plasticity (Cambridge University Press, Cambridge, 2002).

[70] G. B. Ermentrout and N. Kopell, Proc. Natl. Acad. Sci. USA 95, 1259 (1998).

[71] A. Pikovsky, M. Rosenblum, and J. Kurths, Synchronization: A Universal Concept in Nonlinear Sciences (Cambridge University Press, Cambridge, 2003).

[72] C. Börgers, S. Epstein, and N. J. Kopell, Proc. Natl. Acad. Sci. USA 102, 7002 (2005).

[73] A. D. Reyes and E. E. Fetz, J. Neurophysiol. 69, 1661 (1993).

[74] T. I. Netoff, M. I. Banks, A. D. Dorval, C. D. Acker, J. S. Haas, N. Kopell, and J. A. White, J. Neurophysiol. 93, 1197 (2005).

[75] S. Wang, M. M. Musharoff, C. C. Canavier, and S. Gasparini, J. Neurophysiol. 109, 2757 (2013).

[76] R. F. Galán, G. B. Ermentrout, and N. N. Urban, Phys. Rev. Lett. 94, 158101 (2005).
[77] M. Lengyel, J. Kwag, O. Paulsen, and P. Dayan, Nat. Neurosci. 8, 1677 (2005).

[78] K. M. Stiefel, B. S. Gutkin, and T. J. Sejnowski, PLoS One 3, e3947 (2008).

[79] J. G. Mancilla, T. J. Lewis, D. J. Pinto, J. Rinzel, and B. W. Connors, J. Neurosci. 27, 2058 (2007).

[80] L. Glass and M. C. Mackey, From Clocks to Chaos: The Rhythms of Life (Princeton University Press, Princeton, 1988).

[81] T. Gloveli, T. Dugladze, S. Saha, H. Monyer, U. Heinemann, R. D. Traub, M. A. Whittington, and E. H. Buhl, J. Physiol. 562, 131 (2005).

[82] N. Hájos, J. Pálhalmi, E. O. Mann, B. Németh, O. Paulsen, and T. F. Freund, J. Neurosci. 24, 9127 (2004).

[83] J. Csicsvari, B. Jamieson, K. D. Wise, and G. Buzsáki, Neuron 37, 311 (2003).

[84] N. Brunel and X. J. Wang, J. Neurophysiol. 90, 415 (2003).

[85] C. Van Vreeswijk, L. Abbott, and G. B. Ermentrout, J. Comput. Neurosci. 1, 313 (1994).

[86] M. Oh and V. Matveev, J. Comput. Neurosci. 26, 303 (2009).

[87] C. C. Canavier, S. Wang, and L. Chandrasekaran, Front. Neural Circuits 7, 194 (2013).

[88] A. Di Garbo, A. Panarese, M. Barbi, and S. Chillemi, Neurocomputing 70, 2705 (2007).

[89] M. Nomura and T. Aoyagi, Prog. Theor. Phys. 113, 911 (2005).

[90] B. Ermentrout, Neural Comput. 15, 2483 (2003).

[91] C. Leibold, Phys. Rev. Lett. 93, 208104 (2004).

[92] Y. D. Sato and M. Shiino, Phys. Rev. E 66, 041903 (2002).

[93] Q. Wang, Q. Lu, and G. Chen, Int. J. Bifurcat Chaos 18, 1189 (2008).

[94] U. Ernst, K. Pawelzik, and T. Geisel, Phys. Rev. Lett. 74, 1570 (1995).

[95] L. Neltner, D. Hansel, G. Mato, and C. Meunier, Neural Comput. 12, 1607 (2000).

[96] M. Dhamala, V. K. Jirsa, and M. Ding, Phys. Rev. Lett. 92, 074104 (2004).

[97] S. R. Jones, D. J. Pinto, T. J. Kaper, and N. Kopell, J. Comput. Neurosci. 9, 271 (2000).

[98] M. A. Kramer, A. K. Roopun, L. M. Carracedo, R. D. Traub, M. A. Whittington, and N. J. Kopell, PLoS Comput. Biol. 4, e1000169 (2008).

[99] S. Lee, K. Sen, and N. Kopell, PLoS Comput. Biol. 5, e1000602 (2009).

[100] S. Jahnke, R.-M. Memmesheimer, and M. Timme, Front. Comput. Neurosci. 3, 13 (2009).

[101] J. R. Geiger, J. Lübke, A. Roth, M. Frotscher, and P. Jonas, Neuron 18, 1009 (1997).

[102] M. Bartos, I. Vida, M. Frotscher, A. Meyer, H. Monyer, J. R. Geiger, and P. Jonas, Proc. Natl. Acad. Sci. USA 99, 13222 (2002).

[103] M. C. Angulo, J. Rossier, and E. Audinat, J. Neurophysiol. 82, 1295 (1999).

[104] M. T. Craig and C. J. McBain, J. Neurosci. 35, 3616 (2015).

[105] F. E. N. LeBeau, S. K. Towers, R. D. Traub, M. A. Whittington, and E. H. Buhl, J. Physiol. 542, 167 (2002). 